\title{
Tempered Fractional LES Modeling
}

\author{
Mehdi Samiee $^{1,2}$, Ali Akhavan-Safaei ${ }^{1,2}$, and Mohsen Zayernouri ${ }^{1,3} \dagger$ \\ ${ }^{1}$ Department of Mechanical Engineering, Michigan State University, East Lansing, MI 48824, USA \\ ${ }^{2}$ Department of Computational Mathematics, Science and Engineering, Michigan State University, East \\ Lansing, MI 48824, USA \\ ${ }^{3}$ Department of Statistics and Probability, Michigan State University, East Lansing, MI 48824, USA
}

(Received xx; revised xx; accepted xx)

The presence of nonlocal interactions and intermittent signals in the homogeneous isotropic turbulence grant multi-point statistical functions a key role in formulating a new generation of large-eddy simulation (LES) models of higher fidelity. We establish a tempered fractionalorder modeling framework for developing nonlocal LES subgrid-scale models, starting from the kinetic transport. We employ a tempered Lévy-stable distribution to represent the source of turbulent effects at the kinetic level, and we rigorously show that the corresponding turbulence closure term emerges as the tempered fractional Laplacian, $(\Delta+\lambda)^{\alpha}(\cdot)$, for $\alpha \in(0,1), \alpha \neq \frac{1}{2}$, and $\lambda>0$ in the filtered Navier-Stokes equations. Moreover, we prove the frame invariant properties of the proposed model, complying with the subgrid-scale stresses. To characterize the optimum values of model parameters and infer the enhanced efficiency of the tempered fractional subgrid-scale model, we develop a robust algorithm, involving two-point structure functions and conventional correlation coefficients. In an a priori statistical study, we evaluate the capabilities of the developed model in fulfilling the closed essential requirements, obtained for a weaker sense of the ideal LES model (Meneveau 1994). Finally, the model undergoes the a posteriori analysis to ensure the numerical stability and pragmatic efficiency of the model.

\section{Introduction}

With the recent notable developments in computer technologies and, by extension, in the computational mechanics, there is a rapidly growing interest toward using large eddy simulations (LES) in a wide range of applications. Over the past decade, LES modeling has received an increasing attraction from scientific communities as a powerful and promising tool in connection with turbulence phenomena (Piomelli 2014; Bouffanais 2010). In LES, one resolves the large energy-containing eddies by modeling the interplay between large and subgrid scale motions. Due to the tendency of small scales to homogeneous and universal dynamics, LES offers more accurate predictions comparing with the results of resolving the Reynolds-averaged Navier-Stokes (RANS) equations (Holgate et al. 2019; Zhiyin 2015). Furthermore, it lightens the burden of computational costs imposed by accurately capturing the dissipative scales, which renders LES more affordable than direct numerical simulations (DNS).

Concurrent with the recent computational advancements, a marked shift occurred toward using artificial intelligence (AI) as an effective and tractable tool in turbulence modeling due to their significant capabilities in discovering anomalous structures and reproducing nonlocal statistical properties (Beck \& Kurz 2020). In this paradigm, turbulence modeling comes into two divisions: Machine learning based approaches: They introduce advancements in prediction capabilities and reconstructing turbulence structures. Several assorted machine learning (ML) algorithms were proposed for turbulence closure problems including kernel regression and a deep neural network

$\dagger$ Email address for correspondence: zayern@msu.edu 
(Pawar et al. 2020; Sirignano et al. 2020; Portwood et al. 2021). Essentially, pure machine learning based approaches are limited by the representativeness training dataset though they appear to be simpler for implementation. Moreover, to pinpoint complex patterns, large volumes of data are required for the algorithms to learn physical constraints (e.g., frame invariance) and statistical properties, which secondarily makes further complications like optimizing of data compression (Chao et al. 2020). This reveals the significance of physics based models in mentoring the AI approaches and pushing hybrid models as a new direction (Taghizadeh et al. 2020; Willard et al. 2020; Jouybari et al. 2020; Patra et al. 2018), which exploit ML algorithms with a significant reduction in the required input data.

Physics based approaches: They introduce a mathematical representation of physical structures through a number of parameters with a sufficient amount of information. Contrary to ML based approaches, physics based models do not involve large volumes of data although they are inherently limited by the model incompleteness or the complexity of parameterizing physical structures (Chao et al. 2020). Accordingly, it is markedly essential to entail the underlying statistical properties in formulating and inferring an optimum model in a numerically rigorous framework, which links a variety of research disciplines like turbulence, numerical and statistical analysis, and data science. This approach employs principles of physics and borrows insights from the statistical analysis to form a model for real phenomena, which can also be used to guide the ML algorithms (see e.g., You et al. 2021; Kurz \& Beck 2020; Akhavan-Safaei et al. 2020a).

Establishment of such a physically-consistent LES model ties strongly with characterization of nonlocal turbulence mechanisms and a better understanding of anomalous structures. As a puzzling feature, the non-Gaussian behavior of turbulent dynamics is linked to the spatial intermittency of small-scale motions, which is embodied in the form of very thin and elongated vortices (Vincent \& Meneguzzi 1991; Laval et al. 2001). Technically, the nonlocal closure of Navier-Stokes (NS) equations, originated from the Green's function of the Laplacian operator for solving Poisson pressure equation, induces long-range interactions (nonlocal triadic structures) in spectral space of homogeneous turbulence (Sagaut \& Cambon 2008). In a preliminary investigation of isotropic turbulence (She et al. 1990), the significant role of highly vortical structures, typically tube-like, was disclosed on generating nonlocal dynamics and coherence of turbulence. Supported by (Laval et al. 2001), nonlocality as a crucial element in generating intermittent structures has tendency to prevail the local interactions by orders of magnitude. She and Leveque attempted to express self-similar structures in terms of a squence of moment ratios for the energy dissipation field (She \& Leveque 1994). Recently, Mishra and Girimaji (Mishra \& Girimaji 2019) studied the role of pressure on nonlocal mechanisms in incompressible turbulent flows and identified the intercomponent of energy transfer by the rapid pressure strain correlation. For more information, the reader is referred to (Buaria et al. 2020; Pang et al. 2020; Akhavan-Safaei et al. 2020b; Hamlington \& Dahm 2008).

From this perspective, an ideal subgrid-scale (SGS) model represent correctly statistics of the filtered real turbulence at the resolved levels. Given the dependence of an ideal model on an infinite-dimensional set of multi-point statistics, it would be more practical to define a weaker set of conditions in study of SGS parameterization (Sagaut \& Cambon 2008). As one of the earliest studies on statistical analysis of LES, Meneveau (1994) derived a closed set of necessary, yet mild, conditions to fulfill a priori consistency in SGS quantities. More generally, the Karman-Howarth (KH) theorem for anisotropic turbulent flows were studied in (Hill 2002) by eliminating pressure velocity correlations to determine the two-point structure function equations. By proposing a hyper-eddy viscosity term in (Cerutti et al. 2000), SGS dissipation spectrum were measured in locally isotropic turbulence to assess ability of classical two-point closures in prediction of the mean energy transfer. Recently, some of the prevailed challenges in developing an optimal LES model was reviewed succinctly by (Moser et al. 2021). This review presents a clear set of 
statistical characteristics in performing an a priori analysis and providing adequate information for optimizing SGS models.

Statistical descriptions of an ideal closure model derive a desire for developing nonlocal approaches in terms of two-point high-order structure functions in a rigorous mathematical framework. The eddy damped quasi-normal Markovian (EDQNM) approach, described in (Briard et al. 2016), undertakes closing of SGS motions in spectral space by involving high-order statistical moments. As a functional approach, direct interaction approximation pushes the nonMarkovanized stochastic models to the direction of turbulence closure problem, whose solutions are constructed in a fraction form (Shivamoggi \& Tuovila 2019). Furthermore, multifractal models (Yang \& Lozano-Durán 2017; Burton \& Dahm 2005) suggest a potential realizable strategy to accurately capture anomalous scaling exponents, observed in turbulent velocity increments. In addressing statistical local and nonlocal interactions, this progress proceeds with modeling turbulent effects at the kinetic level. Premnath et al. (2009) developed a framework for applying dynamic procedure in the lattice-Boltzmann method for LES of inhomogeneous and anisotropic turbulent flows. A new collision approach were proposed by (Jacob et al. 2018) for LES of weakly compressible flows using two forms of the modified Bhatanagar-Gross-Krook (BGK) collision operators. For more comprehensive review of the literature, we refer the reader to (Jin et al. 2018; Sagaut 2010).

Focusing on the key ideas of $(i)$ describing of anomalous structures in turbulence and (ii) nonlocal closure modeling, fractional calculus appears to be a tractable mathematical tool due to their power-law or logarithmic types of kernel. As an alternative approach to standard methods, they leverage their inherent potentials in representing long-range interactions, selfsimilar structures, sharp peaks, and memory effects in a variety of application (see Burkovska et al. 2020; Kharazmi \& Zayernouri 2019; Zayernouri \& Karniadakis 2013). Egolf \& Hutter (2017) generalized Reynolds shear stresses in local zero-equation to the fractional counterparts. Furthermore, Epps and Cushmann-Roisin derived fractional NS equations from the Boltzmann transport equation in (Epps \& Cushman-Roisin 2018), which supply profound understanding of turbulent nonlocal effects at the kinetic level. For more information, Egolf \& Hutter (2020) provided a comprehensive overview of fractional and nonlocal turbulence, spanning from coherent structures to state-of-the-art ideas on closure modeling in canonical flows. Recently, Di Leoni et al. (2020) contributed in fractional LES modeling by developing a two-point correlation based model in a robust physically-meaning framework.

In the class of nonlocal models, Samiee et al. (2020a) laid out a mathematical framework for developing fractional models, which starts treating turbulence effects at the kinetic level. In a precise derivation, the proposed distribution function in the closed form of filtered collision operator, turns into a fractional model in the LES equations. Throughout a data-driven approach, Akhavan-Safaei et al. (2020a) extended the fractional modeling to the LES of scalar turbulence using two-point correlation functions between the SGS scalar flux and filtered scalar gradient.

In the specific case of isotropic turbulent flows, cascading of energy from large to small scales expresses a self-similar behavior in the inertial range, and then it falls exponentially into the dissipation range. Inspired by such real physics phenomena, we focus on developing a nonlocal model by employing a tempered heavy-tailed distribution within the proposed fractional framework, which contributes in tempered fractional SGS (TFSGS) modeling. Such a tractable fractional operator offers a great flexibility in characterizing nonlocal structures in the turbulent inertial and dissipation ranges through fractional and tempering parameters. To achieve the enhanced performance of the proposed model, we also present an optimization algorithm, involving two-point structure functions. Regarding the best approximation of an ideal physics based model, the optimized TFSGS model restores many essential statistical properties of SGS stresses and presents an a priori consistency in the dissipation spectrum.

The paper is organized as follows. In section 2, we introduce some preliminaries of tempered 
fractional calculus. We outline a mathematical framework in section 3 to develop the tempered fractional model from the Boltzmann transport equation and derive the corresponding forms for SGS quantities. Within a statistical framework, we present a two-point structure based algorithm to infer the optimal behavior of the tempered fractional model in section 4. Using the DNS database of an stationary isotropic turbulent flow, we evaluate the statistical a priori analysis and perform a comparative study on the two-point structure functions in section 5. Moreover, we study numerical stability of the LES solutions through an a posteriori investigation in section 5 . Lastly, section 6 summarize the findings with a conclusion.

\section{Preliminaries on Tempered Fractional Calculus}

Fractional calculus introduces well-established mathematical tools for an accurate description of anomalous phenomena, ubiquitous in a wide range of applications from bio-tissues (Naghibolhosseini \& Long 2018; Ionescu et al. 2017) and material science (Suzuki \& Zayernouri 2020; Suzuki et al. 2021; Meral et al. 2010) to vibration (Suzuki et al. 2020), porous media (Samiee et al. 2020b; Zaky et al. 2020; Xie \& Fang 2019) and turbulence (Akhavan-Safaei et al. 2020a; Di Leoni et al. 2020; Epps \& Cushman-Roisin 2018). As alternative approaches to the standard nonlinear models, fractional models offer a great potential in capturing heavy-tailed distributions, self-similar structures, nonlocal interactions, and memory effects. This potential is substantially indicated by power-law or logarithmic kernels of convolution type in the corresponding fractional operators. From the stochastic point of view, fractional transport models arise from the heavytailed distribution functions in modeling the underlying super- or sub-diffusive motions of particles in complex heterogeneous systems at the microscopic level (Samiee et al. 2019). Nevertheless, common patterns in nature follow finite variance dynamics, which urges the role of tempered fractional calculus as a more sophisticated approach in representing natural cut-offs in real applications and retaining their finite statistical properties.

Recalling from (Sabzikar et al. 2015; Zayernouri et al. 2015), we begin by the definitions of the left- and right-sided tempered fractional derivatives respectively as

$$
\mathcal{D}_{ \pm x}^{\alpha, \lambda} u(x)=\frac{\alpha}{\Gamma(1-\alpha)} \int_{0}^{\infty} \frac{u(x)-u(x \mp s)}{s^{\alpha+1} e^{\lambda s}} d s,
$$

where the fractional derivative order, $\alpha \in(0,1)$, and the tempering parameter, $\lambda>0$. Also, $\Gamma(\cdot)$ represents a Gamma function. For $\alpha \in(1,2)$, the corresponding fractional derivatives are given by:

$$
\mathcal{D}_{ \pm x}^{\alpha, \lambda} u(x)=\frac{\alpha(\alpha-1)}{\Gamma(2-\alpha)} \int_{0}^{\infty} \frac{u(x \mp s)-u(x) \pm s \frac{d u(x)}{d x}}{s^{\alpha+1} e^{\lambda s}} d s .
$$

The link between the derivatives in (2.2) and (2.3) and their counterparts in the Riemann-Liouville sense are described by

$$
\begin{aligned}
{ }^{R L} \mathcal{D}_{ \pm x}^{\alpha, \lambda} u(x) & =\mathcal{D}_{ \pm x}^{\alpha, \lambda} u(x)+\lambda^{\alpha} u(x) \\
{ }^{R L} \mathcal{D}_{ \pm x}^{\alpha, \lambda} u(x) & =\mathcal{D}_{ \pm x}^{\alpha, \lambda} u(x)+\lambda^{\alpha} u(x) \pm \alpha \lambda^{\alpha-1} \frac{d u(x)}{d x} .
\end{aligned}
$$

In particular, for $n \geqslant 0$ the tempered integer-order derivatives are reduced as

$$
{ }^{R L} \mathcal{D}_{+x}^{n, \lambda} u(x)=e^{-\lambda x} \frac{d^{n}\left(e^{\lambda x} u(x)\right)}{d x^{n}},
$$

which recover the classic integer-order derivatives as $\lambda \rightarrow 0$.

Let $\mathcal{F}[u](\xi)$ denote the Fourier transform of $u$, where $\xi$ is the Fourier numbers. Then, we 
obtain

$$
\mathcal{F}\left[R L \mathcal{D}_{ \pm x}^{\alpha, \lambda} u(x)\right]=(\lambda \pm \mathfrak{i} \xi)^{\alpha} \mathcal{F}[u](\xi) .
$$

In this context, the corresponding Fourier transform of the left- and right-sided tempered fractional integrals are given by

$$
\mathcal{F}\left[R L^{2} \mathcal{I}_{ \pm x}^{\alpha, \lambda} u(x)\right](\xi)=(\lambda \pm \mathfrak{i} \xi)^{-\alpha} \mathcal{F}[u](\xi) .
$$

Evidently, tempered integrals and derivatives functions as inverse operators when $u$ possesses sufficient regularity (see Zhang et al. 2018; Sabzikar et al. 2015). Moreover, tempered fractional operators preserve semi-group property, which prepares a useful and rigorous framework for further numerical considerations.

\subsection{Tempered fractional Laplacian}

Denoted by $(\Delta+\lambda)^{\alpha}(\cdot)$, we define the tempered fractional Laplacian of the integral form as

$$
(\Delta+\lambda)^{\alpha} u(\boldsymbol{x})=C_{d, \alpha} \mathrm{P} . \mathrm{V} \cdot \int_{\mathbb{R}^{d}} \frac{u(\boldsymbol{x})-u(\boldsymbol{s})}{e^{\lambda|\boldsymbol{x}-\boldsymbol{s}|}|\boldsymbol{x}-\boldsymbol{s}|^{2 \alpha+d}} d \boldsymbol{s},
$$

where $C_{d, \alpha}=\frac{-\Gamma\left(\frac{d}{2}\right)}{2 \pi^{\frac{d}{2}} \Gamma(-2 \alpha)} \frac{1}{{ }_{2} F_{1}\left(-\alpha, \frac{d+2 \alpha-1}{2} ; \frac{d}{2} ; 1\right)}$ for $\alpha \in(0,1)$ and $\alpha \neq \frac{1}{2}$. In particular, for $d=1$ $(\Delta+\lambda)^{\alpha}$ is reduced to the so-called Riesz fractional form, described by

$$
\begin{aligned}
(\Delta+\lambda)^{\alpha} u(x) & =(-1)^{\lfloor 2 \alpha\rfloor+1} \frac{R L}{\mathcal{D}_{+x}^{\alpha, \lambda} u(x)+{ }^{R L} \mathcal{D}_{-x}^{\alpha, \lambda} u(x)} \\
& =C_{\alpha} \text { P.V. } \int_{\mathbb{R}} \frac{u(x)-u(s)}{e^{\lambda|x-s|}|x-s|^{2 \alpha+1}} d s,
\end{aligned}
$$

where $C_{\alpha}=\frac{-\Gamma\left(\frac{1}{2}\right)}{2 \pi^{\frac{1}{2}} \Gamma(-2 \alpha)} \frac{1}{\cos (\pi \alpha)}$ (see Zhang et al. 2018). In Appendix A, we detail the derivation of the Fourier transform of $(\Delta+\lambda)^{\alpha} u(\boldsymbol{x})$, formulated as

$$
\begin{aligned}
\mathcal{F}\left[(\Delta+\lambda)^{\alpha} u(\boldsymbol{x})\right](\boldsymbol{\xi})= & \mathfrak{C}_{d, \alpha} \times \\
& \left(\lambda^{2 \alpha}-\left(\lambda^{2}+\xi^{2}\right)^{\alpha}{ }_{2} F_{1}\left(-\alpha, \frac{d+2 \alpha-1}{2} ; \frac{d}{2} ; \frac{\xi^{2}}{\xi^{2}+\lambda^{2}}\right)\right) \mathcal{F}[u](\boldsymbol{\xi}),
\end{aligned}
$$

in which $\mathfrak{C}_{d, \alpha}=\frac{1}{{ }_{2} F_{1}\left(-\alpha, \frac{d+2 \alpha-1}{2} ; \frac{d}{2} ; 1\right)}$ and $\xi=|\xi|$. For $d=3$, we define $\mathfrak{C}_{\alpha}=\frac{1}{{ }_{2} F_{1}\left(-\alpha, \frac{2+2 \alpha}{2} ; \frac{3}{2} ; 1\right)}$. It is worth noting that when $\lambda$ approaches 0 , we recover the usual fractional Laplacian in both integral or Fourier forms.

\section{Boltzmannian Framework}

The kinetic Boltzmann transport (BT) is a formal framework for describing fluid particle motions over a wide range of flow physics (e.g., rarefied gas flows and turbulence). This framework offers a great potential for statistical description of turbulent small-scales towards a better understanding of coherent structures in turbulence yet at the kinetic level. As an alternative approach in turbulent closure modeling, reconciling SGS terms in the BT and the Navier-Stokes (NS) equations can conceivably give rise to a rigorous physics based model at the continuum level.

Within the BT framework proposed in (Samiee et al. 2020a), we develop a SGS model, respecting the statistical and physical properties of turbulent unresolved-scale motions. 


\subsection{Subgrid-scale modeling}

In the description of incompressible turbulent flows, we consider large eddy simulation (LES) equations (Pope 2001), governing the dynamics of the resolved-scale flow variables,

$$
\frac{\partial \overline{\boldsymbol{V}}}{\partial t}+\overline{\boldsymbol{V}} \cdot \nabla \overline{\boldsymbol{V}}=-\frac{1}{\rho} \nabla \bar{p}+v \nabla \cdot \overline{\boldsymbol{S}}-\nabla \cdot \boldsymbol{T}^{\mathcal{R}},
$$

where in the index form $\overline{\boldsymbol{V}}(\boldsymbol{x}, t)=\bar{V}_{i}$ and $\bar{p}(\boldsymbol{x}, t)$ represent the velocity and the pressure fields for $i=1,2,3$ and $\boldsymbol{x}=x_{i}$. Moreover, $v$ and $\rho$ denote the kinematic viscosity and the density, respectively. Considering $\mathcal{L}$ as the filter width, the filtered field is obtained in the form of $\overline{\boldsymbol{V}}=G * \boldsymbol{V}$, where $G=G(\boldsymbol{x})$ denotes the kernel of a spatial isotropic filtering type and $*$ is the convolution operator. By implementing the filtering operation, we decomposes the velocity field, $\boldsymbol{V}$, into the filtered (resolved), $\overline{\boldsymbol{V}}$, and the residual, $\boldsymbol{v}$, components. In (3.1), the filtered strain rate, $\overline{\boldsymbol{S}}$, and the SGS stress tensor, $\boldsymbol{T}^{\mathcal{R}}$, are defined by $\bar{S}_{i j}=\frac{1}{2}\left(\frac{\partial V_{i}}{\partial x_{j}}+\frac{\partial V_{j}}{\partial x_{i}}\right)$ and $T_{i j}^{\mathcal{R}}=\overline{V_{i} V_{j}}-\bar{V}_{i} \bar{V}_{j}$.

Since the filtering operator cannot commute with the nonlinear terms in the NS equations, SGS stresses must be modeled in terms of the resolved velocity field. As a common yet reliable approach, Smagorinsky (Smagorinsky 1963) offered modeling the SGS stresses borrowing the Boussinessq approximation from the kinetic theory such that $\boldsymbol{T}^{\mathcal{R}}=-2 v_{R} \overline{\boldsymbol{S}}$ and $v_{R}$ is indicated by $v_{R}=\left(C_{s} \mathcal{L}\right)^{2}|\overline{\boldsymbol{S}}|$, where $|\overline{\boldsymbol{S}}|=\sqrt{2 \bar{S}_{i j} \bar{S}_{i j}}$ and $C_{s}$ is the Smagorinsky (SMG) constant.

\subsection{The BGK equation and the closure problem}

Starting from the Boltzmann kinetic theory (Soto 2016), the evolution of mass distribution function $f$ is governed by the Boltzmann transport (BT) equation as

$$
\frac{\partial f}{\partial t}+\boldsymbol{u} \cdot \nabla f=\Omega(f),
$$

in which $f(t, \boldsymbol{x}, \boldsymbol{u}) d \boldsymbol{x} d \boldsymbol{u}$ represent the probability of finding mass of particles, located within volume $d \boldsymbol{x} d \boldsymbol{u}$ centered on a specific location, $\boldsymbol{x}$, and speed, $\boldsymbol{u}$, at time $t$. It is worth noting that in the particle phase space $\boldsymbol{x}, \boldsymbol{u}$, and $t$ are independent variables. Technically, the left-hand side of (3.2) concerns the streaming of non-reacting particles in absence of any body force and the right-hand side represent the collision operator. The most common form of $\Omega(f)$ with a single collision is the so-called BGK approximation (Soto 2016), given by

$$
\Omega(f)=-\frac{f-f^{e q}}{\tau},
$$

where $\tau$ represent the single relaxation time. In the case of incompressible flows with a roughly constant temperature, $\tau$ is assumed to be independent of macroscopic flow field velocity and pressure. Moreover, under the circumstances of thermodynamic equilibrium of particles, $f^{e q}(\Delta)$ serves as

$$
f^{e q}(\Delta)=\frac{\rho}{U^{3}} F(\Delta)
$$

where $F(\Delta)=e^{-\Delta / 2}, \Delta=\frac{|\boldsymbol{u}-\boldsymbol{V}|^{2}}{U^{2}}$ as an isotropic Maxwellian distribution and $U$ denotes the agitation speed. More specifically, $U=\sqrt{3 k_{B} T / m}$, in which $k_{B}, T$, and $m$ represent the Boltzmann constant, room temperature, and the molecular weight of air.

By recalling the basics of BT equation from (Epps \& Cushman-Roisin 2018; Samiee et al. $2020 a$ ), we introduce the following quantities: $L$ as the macroscopic length scale, $l_{s}$ as the microscopic characteristic length associated with the Kolmogorov length scale, $l_{m}$ as the average distance, traveled by a particle between successive collisions. Furthermore, we define $\boldsymbol{x}^{\prime}$ as the location of particles before scattering, where $\boldsymbol{x}$ is the current location. Thus, $\boldsymbol{x}^{\prime}=\boldsymbol{x}-\left(t-t^{\prime}\right) \boldsymbol{u}$, 
where $\boldsymbol{u}$ is assumed to be constant during $t-t^{\prime}$. The analytical solution of (3.2) and (3.3) is given by

$$
\begin{aligned}
f(t, \boldsymbol{x}, \boldsymbol{u}) & =\int_{0}^{\infty} e^{-s} f^{e q}(t-s \tau, \boldsymbol{x}-s \tau \boldsymbol{u}, \boldsymbol{u}) d s \\
& =\int_{0}^{\infty} e^{-s} f_{s, s}^{e q}(\Delta) d s,
\end{aligned}
$$

where $s \equiv \frac{t-t^{\prime}}{\tau}$ and $f_{s, s}^{e q}(\Delta)=f^{e q}(t-s \tau, \boldsymbol{x}-s \tau \boldsymbol{u}, \boldsymbol{u})$.

REMARK 1. In order to develop an LES model within the kinetic transport framework, we constrain our attention to the BT equation with the BGK collision approximation, involving a single relaxation time. Moreover, we follow Assumption 1 in (Samiee et al. 2020a, pp. 4) in the further derivations to establish a physical connection between the collision operator and the convective terms at the continuum level.

In description of turbulence effects at the kinetic level, we decompose $f$ into the filtered, $\bar{f}$, and residual values, $f^{\prime}$, where $\bar{f}=G * f$. As defined previously, $G$ represents the kernel of any generic spatial isotropic filtering type. Then, the filtered kinetic transport for $\bar{f}$ suffices:

$$
\frac{\partial \bar{f}}{\partial t}+\boldsymbol{u} \cdot \nabla \bar{f}=-\frac{\bar{f}-\overline{f^{e q}(\Delta)}}{\tau},
$$

in which $u$ is independent of $t$ and $\boldsymbol{x}$. Ensuing (3.5), the analytical solution of (3.6) is described by

$$
\bar{f}(t, \boldsymbol{x}, \boldsymbol{u})=\int_{0}^{\infty} e^{-s} \overline{f_{s, s}^{e q}(\Delta)} d s
$$

where $\overline{f_{s, s}^{e q}(\Delta)}=\overline{f^{e q}(\Delta(t-s \tau, \boldsymbol{x}-s \tau \boldsymbol{u}, \boldsymbol{u}))}$. Let define $\bar{\Delta}:=\frac{|\boldsymbol{u}-\overline{\boldsymbol{V}}|^{2}}{U^{2}}$. Due to the nonlinear character of the collision operator (Girimaji 2007), the filtering operation does not commute with $\Omega$, which yields the following inequality as

$$
\overline{f^{e q}(\Delta)}=\frac{\rho}{U^{3}} \overline{e^{-\Delta / 2}} \neq \frac{\rho}{U^{3}} e^{-\bar{\Delta} / 2}=f^{e q}(\bar{\Delta}) .
$$

This inequality gives rise to the so-called turbulence closure problem at the kinetic level. From the mathematical standpoint, the SGS motions stem from the convective nonlinear terms in the NS equations, which resembles with the corresponding advective term of the BT equation. Therefore, it seems natural to recognize $\boldsymbol{u} \cdot \nabla f$ responsible for the unresolved turbulence effects in the BT equation, they manifest implicitly via the filtered collision operator though. That is, the filtered collision term in the right-side of (3.6) undertakes not only molecular collisions, but also the embedded SGS motions. By emphasizing on the importance of modeling $\overline{f^{e q}(\Delta)}$ in the filtered collision operator, we review some different approaches in treating nonlinear effects.

Classical approaches: As a common practice in modeling the SGS closures, the attentions were directed toward with eddy-viscosity approximations by employing a modified relaxation time, $\tau^{\star}$, in the BT equation (e.g., Sagaut 2010). Therefore, the proposed filtered BT equation reads as

$$
\frac{\partial \bar{f}}{\partial t}+\boldsymbol{u} \cdot \nabla \bar{f}=-\frac{\bar{f}-f^{e q}(\bar{\Delta})}{\tau^{\star}} .
$$

In this approach, the inequality in (3.8) is disregarded through using $\tau^{\star}$, which renders the SGS model inappropriate for reproducing many features of the SGS motions. Nevertheless, there some non-eddy viscosity models within the lattice Boltzmann framework, which make use of (3.8) to 
propose more consistent SGS model. For more details, the reader is referred to (Chen et al. 2004; Premnath et al. 2009; Malaspinas \& Sagaut 2012).

Fractional approach: In the proposed framework in (Samiee et al. 2020a), the modeling of turbulence nonlinear effects begins with closing the filtered collision operator, where the multiexponential behavior of $\overline{f^{e q}(\Delta)}$ is approximated properly by a heavy-tailed distribution function. Therefore, the $\overline{f^{e q}(\Delta)}$ in (3.6) is described by

$$
\overline{f^{e q}(\Delta)}-f^{e q}(\bar{\Delta}) \simeq f^{\beta}(\bar{\Delta}),
$$

where $f^{\beta}(\bar{\Delta})=\frac{\rho}{U^{3}} F^{\beta}(\Delta)$ and $F^{\beta}(\Delta)$ denotes an isotropic Lévy $\beta$-stable distribution. By taking the first moment of (3.6), one derives the corresponding fractional Laplacian operator, termed as fractional SGS (FSGS) model, at the continuum level, where

$$
\left(\nabla \cdot \boldsymbol{T}^{R}\right)=\mu_{\alpha}(-\Delta)^{\alpha} \overline{\boldsymbol{V}}
$$

where $\mu_{\alpha}=\frac{\rho(U \tau)^{2 \alpha} \Gamma(2 \alpha+1)}{\tau} \frac{2^{2 \alpha} \Gamma(\alpha+d / 2)}{\pi^{d / 2} \Gamma(-\alpha)} \mathrm{c}_{\alpha}$ for $\alpha \in(0,1)$ and $\mathrm{c}_{\alpha}$ is a real-valued constant. In principle, the choice of distribution function in (3.10) gives rise to a nonlocal operator of the resolved flow field in (3.1) as an SGS model.

Despite the notable potentials of the FSGS model in maintaining some important physical and mathematical properties of the SGS stresses, it lacks a finite second-order statistical moment. To control this statistical barrier in the FSGS model and to achieve more congruence between both sides of (3.10), we seek a finite-variance alternative for the Lévy $\beta$-stable distribution by employing the tempered counterpart and thereby a more flexible and predictive fractional operator in the LES equations in the following subsection.

\subsection{Tempered fractional SGS modeling}

Multi-exponential functions express a power-law behavior in the moderate range of distribution and eventually relaxes into an exponential decay (see Evin et al. 2016). By engaging more exponential terms to a multi-exponential function, the corresponding power-law behavior extends toward long ranges; however, it is bound to vanish exponentially at the tail of the distribution, enforced by nature of the physical phenomenon. As a rich class of stochastic functions for fitting into realistic phenomena, tempered stable distributions (Sabzikar et al. 2015) resemble a sheer power-law at the moderate range and then converge to an exponential decay.

Inspired by this argument, we propose to model $\overline{f^{e q}(\Delta)}$ with a coefficient of tempered Lévy $\beta$-stable distribution, denoted by $f^{\beta, \lambda}(\bar{\Delta})$, within the proposed fractional framework as

$$
\overline{f^{e q}(\Delta)}-f^{e q}(\bar{\Delta}) \simeq f^{\text {Model }}(\bar{\Delta})=\mathrm{c}_{\beta, \lambda} f^{\beta, \lambda}(\bar{\Delta}),
$$

where $\mathrm{c}_{\beta, \lambda}$ is a real-valued constant number. Moreover, we consider $\beta \in\left(-1-\frac{d}{2},-\frac{d}{2}\right), \lambda>0$ and $d=3$ represents dimension of the physical domain. Therefore the filtered BT equation reads as

$$
\begin{aligned}
\frac{\partial \bar{f}}{\partial t}+\boldsymbol{u} \cdot \nabla \bar{f} & =-\frac{\bar{f}-f^{e q}(\bar{\Delta})+f^{e q}(\bar{\Delta})-\overline{f^{e q}(\Delta)}}{\tau} \\
& \simeq-\frac{\bar{f}-f^{e q}(\bar{\Delta})-f^{\text {Model }}(\bar{\Delta})}{\tau} .
\end{aligned}
$$

For the sake of simplicity, we take $f^{*}(\bar{\Delta})=f^{e q}(\bar{\Delta})+f^{\text {Model }}(\bar{\Delta})$. The approximation in (3.13) conceivably provides a good fit into the filtered collision operator by maintaining the significant statistical features of $\overline{f^{e q}(\Delta)}$ and sets a physically richer starting point for developing a more expressive nonlocal LES model at the continuum level.

In this regard, the macroscopic variables associated with the flow field can be reconstructed 
according to

$$
\begin{aligned}
\bar{\rho} & =\int_{\mathbb{R}^{d}} \bar{f}(t, \boldsymbol{x}, \boldsymbol{u}) d \boldsymbol{u}, \\
\bar{V}_{i} & =\frac{1}{\rho} \int_{\mathbb{R}^{d}} u_{i} \bar{f}(t, \boldsymbol{x}, \boldsymbol{u}) d \boldsymbol{u}, \quad i=1,2,3,
\end{aligned}
$$

where $\bar{\rho}=\rho$ for an incompressible flow. To establish the connection between the kinetic description and the filtered NS equation, we proceed with deriving the macroscopic form of (3.13) by multiplying it with $\boldsymbol{u}$, and integrating over the kinetic momentum, which yields

$$
\int_{\mathbb{R}^{d}}\left(\boldsymbol{u} \frac{\partial \bar{f}}{\partial t}+\nabla \cdot\left(\boldsymbol{u}^{2} \bar{f}\right)\right) d \boldsymbol{u}=0 \quad \Longrightarrow \quad \rho \frac{\partial \overline{\boldsymbol{V}}}{\partial t}+\nabla \cdot \int_{\mathbb{R}^{d}} \boldsymbol{u}^{2} \bar{f} d \boldsymbol{u}=0 .
$$

Recalling the assumptions in Remark 1 that $\int_{\mathbb{R}^{d}} \boldsymbol{u}\left(\frac{\bar{f}-f^{*}(\bar{\Delta})}{\tau}\right) d \boldsymbol{u}=0$ due to microscopic reversibility of particle collisions. Following the derivations in (Samiee et al. 2020a, pp. 5-6), we add and subtract $\overline{\boldsymbol{V}} \overline{\boldsymbol{V}}$ to the advection term and accordingly, (3.16) is found to be

$$
\rho\left(\frac{\partial \overline{\boldsymbol{V}}}{\partial t}+\nabla \cdot \overline{\boldsymbol{V}} \overline{\boldsymbol{V}}\right)=-\nabla \cdot \varsigma
$$

where $\varsigma$ in the index form is expressed as

$$
\varsigma_{i j}=\int_{\mathbb{R}^{d}}\left(u_{i}-\bar{V}_{i}\right)\left(u_{j}-\bar{V}_{j}\right) \bar{f} d \boldsymbol{u} .
$$

Comparing (3.1) and (3.17), it turns out that pressure term, viscous and SGS stresses all trace back to $\nabla \cdot \varsigma$, where $\varsigma_{i j}=-\bar{p} \delta_{i j}+T_{i j}^{\text {shear }}+T_{i j}^{\mathcal{R}}$. By plugging (3.7) into the kinetic definitions of each term in $\varsigma_{i j}$, we obtain

$$
\begin{aligned}
\bar{p} \delta_{i j} & =-\int_{\mathbb{R}^{d}}\left(u_{i}-\bar{V}_{i}\right)\left(u_{j}-\bar{V}_{j}\right) f^{*}(\bar{\Delta}) d \boldsymbol{u} \int_{0}^{\infty} e^{-s} d s, \\
T_{i j}^{\text {shear }} & =\int_{0}^{\infty} \int_{\mathbb{R}^{d}}\left(u_{i}-\bar{V}_{i}\right)\left(u_{j}-\bar{V}_{j}\right) \times\left(f_{s, s}^{e q}(\bar{\Delta})-f^{e q}(\bar{\Delta})\right) e^{-s} d \boldsymbol{u} d s=2 \mu \bar{S}_{i j},
\end{aligned}
$$

where $\mu=\rho U^{2} \tau$. Similarly, by employing $f^{\text {Model }}$ in (3.12), we attain

$$
\begin{aligned}
T_{i j}^{R} & =\mathrm{c}_{\beta, \lambda} \int_{0}^{\infty} \int_{\mathbb{R}^{d}}\left(u_{i}-\bar{V}_{i}\right)\left(u_{j}-\bar{V}_{j}\right)\left(f_{s, s}^{\beta, \lambda}(\bar{\Delta})-f^{\beta, \lambda}(\bar{\Delta})\right) e^{-s} d \boldsymbol{u} d s \\
& =\frac{\rho \mathrm{c}_{\beta, \lambda}}{U^{3}} \int_{0}^{\infty} \int_{\mathbb{R}^{d}}\left(u_{i}-\bar{V}_{i}\right)\left(u_{j}-\bar{V}_{j}\right)\left(F^{\beta, \lambda}\left(\bar{\Delta}_{s, s}\right)-F^{\beta, \lambda}(\bar{\Delta})\right) e^{-s} d \boldsymbol{u} d s,
\end{aligned}
$$

in which $\bar{\Delta}_{s, s}=\bar{\Delta}(t-s \tau, \boldsymbol{x}-s \tau \boldsymbol{u}, \boldsymbol{u})$. As discussed in (Samiee et al. 2020a, Appendix), the temporal shift can be detached from $f_{s, s}^{\beta, \lambda}(\bar{\Delta})$ and then $\bar{\Delta}_{s, s}$ is simplified to $\bar{\Delta}_{s}=\bar{\Delta}(t, \boldsymbol{x}-s \tau \boldsymbol{u}, \boldsymbol{u})$. Therefore,

$$
T_{i j}^{R}=\frac{\rho \mathrm{c}_{\beta, \lambda}}{U^{3}} \int_{0}^{\infty} \int_{\mathbb{R}^{d}}\left(u_{i}-\bar{V}_{i}\right)\left(u_{j}-\bar{V}_{j}\right)\left(F^{\beta, \lambda}\left(\bar{\Delta}_{s}\right)-F^{\beta, \lambda}(\bar{\Delta})\right) e^{-s} d \boldsymbol{u} d s .
$$

The strategy to evaluate $T_{i j}^{R}$ is to decouple the particle speed into time and displacement by employing $\boldsymbol{u}=\frac{\boldsymbol{x}^{\prime}-\boldsymbol{x}}{\boldsymbol{s} \tau}$ and approximate the asymptotic behavior of $F^{\beta, \lambda}(\bar{\Delta})$ with a tempered power-law distribution. In a detailed discussion in Appendix B, we show that

$$
T_{i j}^{R}=\mathrm{c}_{\alpha, \lambda} \bar{v}_{\alpha} \sum_{k=0}^{\mathcal{K}} \bar{\phi}_{k}^{\mathcal{K}}(\alpha) \int_{\mathbb{R}^{d}}\left(x_{i}-x_{i}^{\prime}\right)\left(x_{j}-x_{j}^{\prime}\right) \frac{\left(\boldsymbol{x}-\boldsymbol{x}^{\prime}\right) \cdot\left(\overline{\boldsymbol{V}}-\overline{\boldsymbol{V}}^{\prime}\right)}{\left|\boldsymbol{x}-\boldsymbol{x}^{\prime}\right|^{2 \alpha+5} e^{\bar{\lambda}_{k}\left|\boldsymbol{x}-\boldsymbol{x}^{\prime}\right|}} d \boldsymbol{x}^{\prime},
$$


in which $\bar{v}_{\alpha}=(2 \alpha+3)\left(\rho C_{\alpha} \tau^{2 \alpha-1} U^{2 \alpha}\right)$ for $\alpha \in\left(0, \frac{1}{2}\right) \cup\left(\frac{1}{2}, 1\right)$ and recalling $\bar{\lambda}_{k}=\frac{k}{\tau U} \lambda$. Moreover, $\bar{\phi}_{k}^{\mathcal{K}}(\alpha)$ is indicated in (B 7). Eventually, we disclose the integral form of $\nabla \cdot \boldsymbol{T}^{\mathcal{R}}$ as

$$
\left(\nabla \cdot \boldsymbol{T}^{\mathcal{R}}\right)_{j}=\mathrm{c}_{\alpha, \lambda} \bar{v}_{\alpha} \sum_{k=0}^{\mathcal{K}} \frac{\left(2 \alpha+\bar{\lambda}_{k}\right)}{(2 \alpha+3)} \bar{\phi}_{k}^{\mathcal{K}}(\alpha) \int_{\mathbb{R}^{d}} \frac{\left(\bar{V}_{j}-\bar{V}_{j}^{\prime}\right)}{\left|\boldsymbol{x}-\boldsymbol{x}^{\prime}\right|^{2 \alpha+3} e^{\bar{\lambda}_{k}\left|\boldsymbol{x}-\boldsymbol{x}^{\prime}\right|}} d \boldsymbol{x}^{\prime},
$$

where $v_{\alpha}=\mathrm{c}_{\alpha, \lambda} \bar{v}_{\alpha}$. Reminding the integral representation of a tempered fractional Laplacian in (2.6), we formulate the divergence of the SGS stresses as follows

$$
\left(\nabla \cdot \boldsymbol{T}^{\mathcal{R}}\right)_{j}=v_{\alpha} \sum_{k=0}^{\mathcal{K}} \phi_{k}^{\mathcal{K}}(\alpha, \lambda)\left(\Delta+\bar{\lambda}_{k}\right)^{\alpha} \bar{V}_{j}
$$

where $\phi_{k}^{\mathcal{K}}(\alpha, \lambda)=\frac{\left(2 \alpha+\bar{\lambda}_{k}\right)}{(2 \alpha+3)} \bar{\phi}_{k}^{\mathcal{K}}(\alpha)$. Evidently, by setting $\mathcal{K}=0$, we find $\bar{\phi}_{k}^{\mathcal{K}}(\alpha)=\Gamma(2 \alpha)$ and the new operator in (3.25) reduces to a fractional Laplacian, which recovers the FSGS model.

REMARK 2. In terms of the explicit Fourier form of the tempered operator, $(\Delta+\bar{\lambda})^{\alpha}(\cdot)$, the TFSGS model maintains the high-order accuracy of scheme in LES solutions similar to the eddy-viscosity models without including any computational cost.

Inferring from (3.25), our choice in the kinetic description of turbulent effects reflects in the form of a tempered fractional operator through a rigorous connection between the filtered BT and NS equations. More specifically, we adopt $\mathcal{K}=1$ and hence the tempered fractional SGS (TFSGS) model can be formulated as

$$
\nabla \cdot \boldsymbol{T}^{\mathcal{R}}=v_{\alpha}\left[\phi_{0}^{1}(\alpha)\left(-(-\Delta)^{\alpha}\right) \overline{\boldsymbol{V}}+\phi_{1}^{1}(\alpha)\left(\Delta+\bar{\lambda}_{1}\right)^{\alpha} \overline{\boldsymbol{V}}\right]
$$

where $\phi_{0}^{1}(\alpha)=\frac{1}{(2 \alpha+3)}(\Gamma(2 \alpha+1)-\Gamma(2 \alpha))$ and $\phi_{1}^{1}(\alpha, \lambda)=\frac{(2 \alpha+\lambda)}{(2 \alpha+3)} \Gamma(2 \alpha-1)$. Accordingly, the governing LES equations read as

$$
\frac{\partial \bar{V}_{i}}{\partial t}+\frac{\partial \bar{V}_{i} \bar{V}_{j}}{\partial x_{j}}=-\frac{1}{\rho} \frac{\partial \bar{p}}{\partial x_{i}}+v \Delta \bar{V}_{i}-v_{\alpha} \sum_{k=0}^{1} \phi_{k}^{1}(\alpha, \lambda)\left(\Delta+\bar{\lambda}_{k}\right)^{\alpha} \bar{V}_{i},
$$

where $\alpha \in\left(0, \frac{1}{2}\right) \cup\left(\frac{1}{2}, 1\right), \lambda>0$, and $v_{\alpha}=\frac{\mu_{\alpha}}{\rho}$.

REMARK 3. As a generator of tempered Lévy stable processes, the tempered fractional Laplacian is proven to be rotationally and Galilean invariant (see Cairoli 2016; Huang 2015; Kaleta \& Sztonyk 2015). Therefore, by having $v_{\alpha}$ and $\phi_{k}^{1}$ as real-valued functions of $\alpha$ and $\lambda$, the TFSGS model also adopts the frame invariance property in a consistent fashion with the SGS stresses.

\subsection{TFSGS formulations for the SGS stresses}

To study the key role of tempering fractional operators in recovering turbulent statistical structures, it is essential to establish a straightforward form of the modeled SGS stresses. Due to some numerical complications in evaluating the integral in (3.24), we settle to proceed with the Fourier representation of the TFSGS model. Employing the definition of $\mathcal{I}^{\alpha}$ ( $\alpha$-Riesz potential) from (Stein 1970), it is possible to verify that

$$
\nabla \cdot \boldsymbol{T}^{R}=(\Delta+\lambda)^{\alpha} \overline{\boldsymbol{V}}=\nabla \cdot \nabla \mathcal{I}^{\alpha=1}\left[v_{\alpha} \sum_{k=0}^{1} \phi_{k}^{1}(\alpha, \lambda)\left(\Delta+\bar{\lambda}_{k}\right)^{\alpha} \overline{\boldsymbol{V}}\right] .
$$


Inspired by (3.28), we introduce $\mathcal{R}_{j}^{\alpha, \lambda}(\cdot)=\nabla_{j} \mathcal{I}^{\alpha=1}(\Delta+\lambda)^{\alpha}(\cdot)$ as a tempered fractional operator such that

$$
T_{i j}^{R}=\frac{v_{\alpha}}{2} \sum_{k=0}^{1} \phi_{k}^{1}(\alpha, \lambda)\left[\mathcal{R}_{j}^{\alpha, \bar{\lambda}_{k}} V_{i}+\mathcal{R}_{i}^{\alpha, \bar{\lambda}_{k}} V_{j}\right],
$$

where $\mathcal{F}\left[I^{\alpha=1}\right]=\frac{1}{\xi^{2}}$ and $\mathcal{F}\left[\nabla_{j}\right](\xi)=-\mathfrak{i} \xi_{j}$ and $\mathfrak{i}$ denotes an imaginary unite. Following (2.7) into (3.29), we find the Fourier form of $\mathcal{R}_{j}^{\alpha, \lambda}$ as

$$
\mathcal{F}\left[\mathcal{R}_{j}^{\alpha, \lambda}\right](\boldsymbol{\xi})=\mathfrak{C}_{\alpha} \frac{-\mathfrak{i} \xi_{j}}{\xi^{2}}\left(\lambda^{2 \alpha}-\left(\lambda^{2}+\xi^{2}\right)^{\alpha}{ }_{2} F_{1}\left(-\alpha, 1+\alpha ; \frac{3}{2} ; \frac{\xi^{2}}{\xi^{2}+\lambda^{2}}\right)\right) .
$$

\section{Statistical Analysis}

In pursuit of an ideal SGS model, nonlinearity induced by the convective terms and nonlocality imparted by the pressure term in the NS equations contribute to a synthetic hierarchy of transport equations and multi-point descriptions of SGS terms, as shown in (Sagaut \& Cambon 2008). The infinitely-extended hierarchical triangle of nonlinearity and nonlocality brings up the idea of indicating a set of weaker, and yet significant, statistical conditions and make the ideal LES model more attainable, as endorsed by (Moser et al. 2021). To identify such statistical features, Meneveau (1994) developed a rigorous framework via a statistical a priori analysis and formulated some sufficient conditions for the assessment of LES models. As one of the candidates for evaluating SGS models, we give a brief review of the argued formulations in (Meneveau 1994) and introduce an optimization strategy, which enables the TFSGS model to correctly generate the requisite statistical conditions.

Hereafter, we consider the following notations in study of the SGS fields. Let $\boldsymbol{T}^{R, D}$ and $\boldsymbol{T}^{R \text {,* }}$ denote the SGS stresses, implied by the true DNS data and the SGS model, respectively. We also take $\boldsymbol{r}$ as the displacement vector between two points in the correlation functions and $\boldsymbol{e}$ denotes the unit vectors of the axes in the Cartesian coordinates. Then, $r=|\boldsymbol{r}|$. As discussed in (Meneveau 1994), performing an ensemble-average of the filtered NS equations offers a set of necessary conditions for an LES simulation to ensure the equality of mean velocity profiles and the second-order moments, listed as:

(a) $\left\langle T_{i j}^{R, D}\right\rangle=\left\langle T_{i j}^{R, *}\right\rangle$,

(b) $\left\langle\bar{V}_{i} T_{i j}^{R, D}\right\rangle=\left\langle\bar{V}_{i} T_{i j}^{R, *}\right\rangle$,

(c) $\left\langle\bar{S}_{i j} T_{i j}^{R, D}\right\rangle=\left\langle\bar{S}_{i j} T_{i j}^{R, *}\right\rangle$,

in which conditions (b) and (c) are inferred from the ensemble-averaged SGS transport equation.

Focusing on the nonlocality axis of the closure triangle for a homogeneous isotropic turbulent (HIT) flow, one obtains the so-called Karman-Howarth (KH) equation as

$$
\left[\frac{\partial}{\partial t}-2 v\left(\frac{\partial^{2}}{\partial r^{2}}+\frac{4}{r} \frac{\partial}{\partial r}\right)\right] B_{L L}(r, t)-\left(\frac{\partial}{\partial r}+\frac{4}{r}\right) B_{L L L}(r, t)=\left(\frac{\partial}{\partial r}+\frac{4}{r}\right) G_{L L L}(r, t),
$$

for sufficiently large $\mathcal{L} \gg \eta$, where $L$ represents the longitudinal direction. Additionally, we denote by $B_{L L}(r, t)=\left\langle\bar{V}_{L}(\boldsymbol{x}, t) \bar{V}_{L}(\boldsymbol{x}+\boldsymbol{r} \cdot \boldsymbol{e}, t)\right\rangle$ and $B_{L L L}(r, t)=\left\langle\left[\bar{V}_{L}(\boldsymbol{x}, t)\right]^{2} \bar{V}_{L}(\boldsymbol{x}+\boldsymbol{r} \cdot \boldsymbol{e}, t)\right\rangle$ the second- and third-order velocity correlation functions, respectively and $G_{L L L}(r, t)=$ $\left\langle T_{L L}^{R}(\boldsymbol{x}, t) \bar{V}_{L}(\boldsymbol{x}+\boldsymbol{r} \cdot \boldsymbol{e}, t)\right\rangle$ refers to the stress-strain correlation function. Technically, the third-order correlation function in (4.1) is subdivided into $B_{L L L}$ stemming from the resolved velocity field and $G_{L L L}(r, t)$ coming from the SGS stresses. It turns out from (4.1) that the SGS model should undergo a correct prediction of $G_{L L L}(r, t)$ to re-generate $B_{L L}$ and $B_{L L L}$ 
accurately. Referring to (Meneveau 1994, pp. 819-820), we arrive at the following equation

$$
\left\langle\left[\bar{V}_{L}(\boldsymbol{x}+\boldsymbol{r} \cdot \boldsymbol{e}, t)-\bar{V}_{L}(\boldsymbol{x}, t)\right]^{3}\right\rangle+6 G_{L L L}(r, t)=6\left\langle\bar{S}_{L L} T_{L L}^{R}\right\rangle r
$$

which exhibits the only sufficient condition for modeling third-order structure in an HIT flow. Therefore, by satisfying the equality of SGS dissipation via conditions (c), modeling $G_{L L L}$ remains the only requisite for capturing the third-order structure functions.

This finding reveals the significance of condition (c), which intrinsically ties with the stressstrain correlation function, represented by $D_{L L}(r, t)=\left\langle\bar{S}_{L L}(\boldsymbol{x}+\boldsymbol{r} \cdot \boldsymbol{e}, t) T_{L L}^{R}\right\rangle$. Using the conversation in (Cerutti et al. 2000, pp. 317), $D_{L L}$ is derived in terms of $G_{L L L}$ as

$$
D_{L L}(r, t)=\frac{7}{2} \frac{d G_{L L L}(r, t)}{d r}+\frac{4 G_{L L L}(r, t)}{r}+\frac{r}{2} \frac{d^{2} G_{L L L}(r, t)}{d r^{2}} .
$$

Emphasizing the role of tempering parameter in modulating the turbulent dissipation range, we therefore adopt $D_{L L}(r, t)$ as a key quantity in optimizing the TFSGS model to address condition (c) and capture the nonlocal structures in (4.2). It must be noted that in evaluating the aforementioned conditions and high-order structures, $\boldsymbol{T}^{R}$ represents either $\boldsymbol{T}^{R, D}$, obtained by filtering the instant DNS database, or $\boldsymbol{T}^{R \text {, }}$, implied by implementing any model to the true resolved velocity field.

\subsection{Optimization strategy}

Devising a robust optimization framework is an inevitable element in predictive fractional and tempered fractional modeling (see Burkovska et al. 2020; Pang et al. 2020). Regarding the given set of conditions for the closure problem, we find conditions (a) and (c) practically crucial in developing an approach for estimating the parameters and coefficient associated with the TFSGS model while condition (b) can be substantially recovered by imposing (4.2), where $\left.G_{L L L}(r, t)\right|_{r=0}=\left\langle\bar{V}_{i} T_{i j}^{R}\right\rangle$. As we learn from the one-point correlation analysis in (Samiee et al. $2020 a$ ) and the further section, correlations between the SGS stresses, obtained by the DNS data and the model, highly rely on $\alpha$ and $\lambda$ in the TFSGS model rather plays a central role in capturing the SGS dissipation energy and nonlocal structure functions. This approach provides the basis for an optimal estimation of the fractional exponents $(\alpha$ and $\lambda)$ by employing the normalized $D_{L L}$ and $\varrho_{i}$, defined in Algorithm 1.

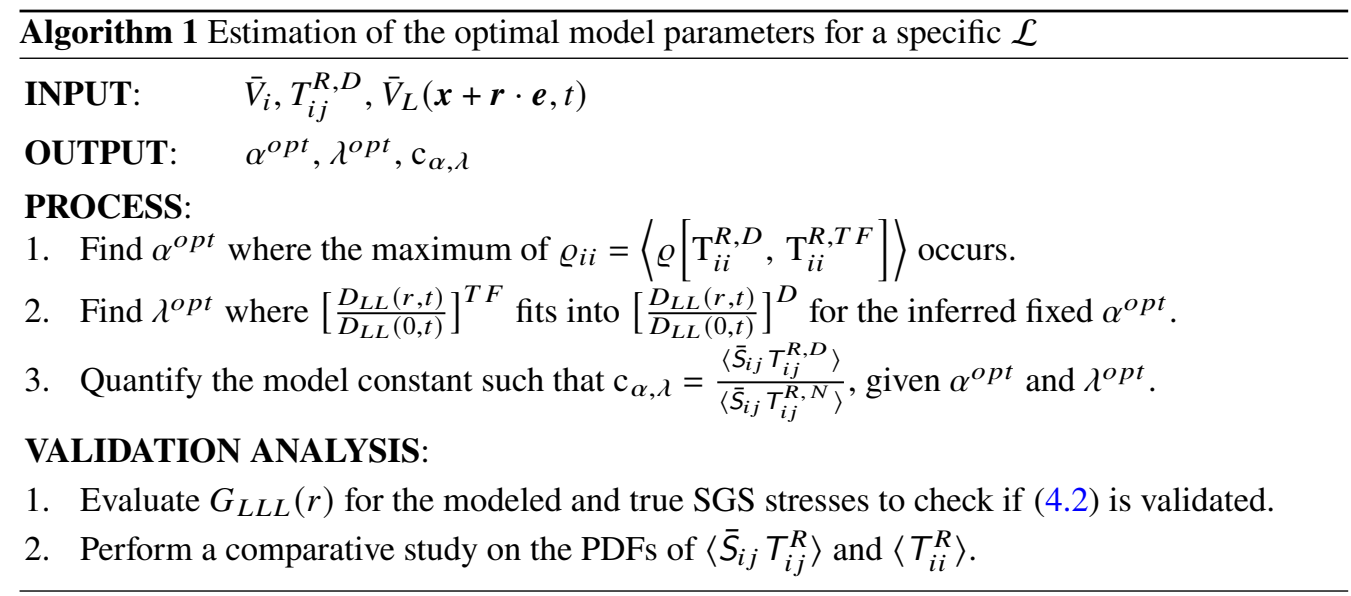

By fixing the values of fractional exponents, it is possible to accurately quantify the model coefficient and thereby re-producing the third-order structure in (4.2) via modeling $G_{L L L}$. In 
Table 1: Flow parameters and statistical properties in the DNS of a forced HIT flow.

\begin{tabular}{cccccccc}
$\operatorname{Re}_{\lambda}$ & $u_{r m s}^{\prime}$ & $K_{\text {tot }}$ & $v$ & $\varepsilon$ & $\tau_{\mathscr{L}}$ & skewness & kurtosis \\
& $(\mathrm{m} / \mathrm{sec})$ & $\left(\mathrm{m}^{2} / \mathrm{sec}^{2}\right)$ & $\left(\mathrm{m}^{2} / \mathrm{sec}\right)$ & $\left(\mathrm{m}^{2} / \mathrm{sec}^{3}\right)$ & $(\mathrm{sec})$ & & \\
\hline 190 & 0.67 & 0.68 & 0.001 & 0.1 & 4.2 & -0.5 & 6.5
\end{tabular}

Algorithm 1, we schematically present the proposed method for optimizing the parameters associated with the TFSGS model at a given flow Reynolds number $(\operatorname{Re})$ and a specific $\mathcal{L}$.

It must be noted that in step 3, we define

$$
T_{i j}^{R, N}=\frac{T_{i j}^{R, T F}}{\mathrm{c}_{\alpha, \lambda}}=\frac{\bar{v}_{\alpha}}{2} \sum_{k=0}^{1} \phi_{k}^{1}(\alpha, \lambda)\left[\mathcal{R}_{j}^{\alpha, \bar{\lambda}_{k}} V_{i}+\mathcal{R}_{i}^{\alpha, \bar{\lambda}_{k}} V_{j}\right] .
$$

Moreover, superscripts " $D$ " and " $T F$ " represent the values obtained by filtering the true DNS data and the TFSGS model, respectively.

\section{A Priori / Posteriori Analyses}

To attain the optimal behavior of the TFSGS model, we follow the steps in Algorithm 1 by performing an a priori analysis and evaluate the capabilities of the TFSGS model in generating the statistical features of turbulent flows.

\subsection{DNS Database and LES Platform}

In terms of a priori tests, we conduct the numerical simulation of a forced HIT flow employing the open-source pseudo-spectral NS solver for a triply periodic domain the code of which is presented at (Akhavan-Safaei \& Zayernouri 2020). It should be noted that in the next section, the LES solver is successfully prepared using this DNS code and the statically stationary DNS dataset presented here is filtered and used as the initial conditions for the final a posteriori assessments.

Using the NS solver, we performed DNS of a stationary HIT flow with $320^{3}$ resolution for a periodic computation domain as $\Omega=[0,2 \pi]^{3}$ and the large scale forcing occurs at $0<|\xi| \leqslant 2$ to maintain turbulence statistics stationary. Here, $\boldsymbol{\xi}$ represents the vector of Fourier wave numbers and $\xi_{\max }=\sqrt{2} N / 3$ is the maximum wave number solved numerically, where $N=320$ is the number of grid points. In this case, $\xi_{\max } \eta_{k}=1.6>1$ certifies that all the scales of motion are well-resolved, where $\eta_{k}$ refers to the Kolmogorov length scale. We detail the flow parameters and some of the statistical properties at Table 1 , in which $\varepsilon$ and $K_{t o t}$ denote the expected values of dissipation rate and turbulent kinetic energy, respectively. Moreover, $R e_{\lambda}=\frac{u_{r m s}^{\prime} l_{\lambda}}{v}$ and $l_{\lambda}=\sqrt{15 v u_{r m s}^{\prime 2} / \varepsilon}$ represent the Taylor Reynolds number and micro-scale length, respectively, where $u_{r m s}^{\prime}=\sqrt{2 K_{\text {tot }} / 3}$. The simulation undergoes running for 30 eddy turn-over times, $\tau_{\mathscr{L}}$, to construct 40 sample snapshots as our database. Due to the present homogeneity and isotropy in the HIT flow, we find the database adequate for obtaining the required statistics in the further analysis. The kurtosis and skewnewss values of the diagonal components of velocity gradient tensor are also presented in Table 1, supporting non-Gaussianity of turbulent structures.

For the purpose of crunching heavy DNS database in the statistical analysis, we develop an LES platform in Python with a focus on efficiency in obtaining two-point correlations and the ease of dealing with the fractional operators. This Platform consists of three chief components: filtering the DNS database, implementation of LES models and optimization, and executing the final 

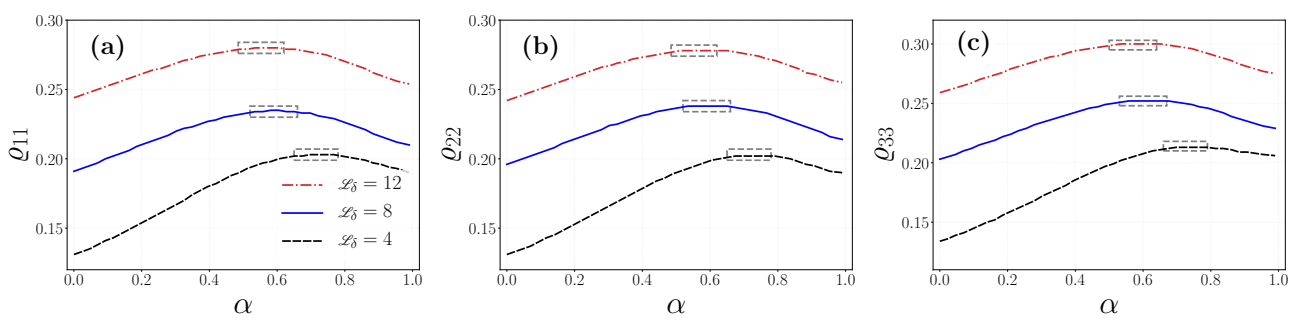

Figure 1: variation of the correlation coefficients (a) $\varrho_{11}$, (b) $\varrho_{22}$, and (c) $\varrho_{33}$ versus $\alpha \in(0,1)$ for $\mathcal{L}_{\delta}=4,8,12$ by setting $\lambda \simeq 0$ in (4.4). The maximum values lie in the dashed boxes.

analysis. To overcome the burden of timely filtering process especially in two-point correlation analysis, we introduce the scalable multi-threaded filtering code using Numpy, threading, and astropy. convolution packages. Further steps in finding the the optimum model parameters and applying the Fourier form of the fractional models are developed employing highly efficient Intel® MKL library.

\subsection{Optimal estimation of fractional parameters}

In order to optimize the efficiency of the TFSGS model, we developed a flexible and rigorous strategy in Algorithm 1. The proposed algorithm is equipped with verification and validation mechanisms through the conventional correlation coefficients and two-point structure functions. Recalling from section 4.1 that $T_{i j}^{R, D}$ denotes the true SGS stresses obtained by filtering the well-resolved DNS data. Moreover, $T_{i j}^{R, *}$ represents the general form of modeled SGS values, where * can be replaced by $T F$ or $S M$ in the TFSGS or SMG models, respectively.

The first step in Algorithm 1 concerns detecting optimum value of fractional exponent, $\alpha^{\text {opt }}$, where the maximum of ensemble-averaged correlation between $T_{i i}^{R, D}$ and $T_{i i}^{R, T F}$, denoted by $\varrho_{i i}$, occurs. Our premise is that the tempering parameter, $\lambda$, does not make any noticeable changes in $\varrho_{i i}$, namely less than 3 percents, which is endorsed by the results of Table 2 . In the absence of $\lambda$, we plot the variations of $\varrho_{i i}$ versus $\alpha^{o p t}$ in Figure 1 for $i=1,2,3$, in which each dashed box specifies the interval of $\alpha$ yielding the maximum of $\varrho_{i i}$. Without any loss of accuracy, we adopt $\alpha^{o p t}=0.76,0.58,0.51$ as the corresponding minimum value in each specified interval for $\mathcal{L}_{\delta}=\frac{\mathcal{L}}{2 \delta x}=4,8,12$, respectively, where $\delta x=\frac{2 \pi}{N}$ represents the computational grid size.

From the kinetic perspective, by enlarging $\mathcal{L}_{\delta}, \overline{f^{e q}(\Delta)}$ in (3.6) demonstrates an increasingly multi-exponential pattern, which can be better described by a power-law distribution function. This argument accounts for the prediction enhancement in Figure 1, achieved by the TFSGS model and the abduct reduction of $\alpha^{o p t}$ versus $\mathcal{L}_{\delta}$ (see Samiee et al. 2020a, page 10). Theoretically, the tempered power-law distribution can resemble a power-law or a Gaussian distribution by letting $\lambda$ go to 0 or $\infty$, respectively. This grounds for the TFSGS model to span the gap between the FSGS model, representing self-similar behavior of the inertial range, and the SMG model, renowned for its dissipative characteristics. The results in Table 2 support this line of reasoning by a row of correlation quantities for the given filter widths, particularly at $\mathcal{L}_{\delta}=12$.

On this background, we proceed with the second step in Algorithm 1 to indicate $\lambda^{o p t}$ through a comparative study of the normalized strain-stress correlation function, defined as $S_{\Delta}=\frac{D_{L L}(r, t)}{D_{L L}(0, t)}$. With the knowledge of $D_{L L}(r, t)$, we extend the two-point correlation analysis to the spectral space by evaluating the instantaneous radial dissipation spectrum, given by $\hat{\mathcal{D}}(\xi)=\mathcal{F}\left[D_{L L}(r, t)\right](\xi)$. To evaluate the error between the dissipation spectrum, obtained by the true DNS data and the 
Table 2: The ensemble-averaged correlation coefficients $\left(\varrho_{i i}\right)$ between SGS stresses obtained by the filtered DNS data $\left(T_{i i}^{R, D}\right)$ and the TFSGS model $\left(T_{i i}^{R, T F}\right)$ for $i=1,2,3$. In the fractional models, $\alpha^{o p t}$ is set as $0.76,0.58$, and 0.51 for $\mathcal{L}_{\delta}=4,8,12$ respectively.

\begin{tabular}{|c|c|c|c|c|c|c|c|c|c|c|c|c|}
\hline \multirow[b]{3}{*}{$\lambda$} & \multicolumn{4}{|c|}{$\mathcal{L}_{\delta}=4$} & \multicolumn{4}{|c|}{$\mathcal{L}_{\delta}=8$} & \multicolumn{4}{|c|}{$\mathcal{L}_{\delta}=12$} \\
\hline & FSGS & TFS & GS & SMG & FSGS & TFS & GS & SMG & FSGS & TFS & GS & SMG \\
\hline & 0 & 0.1 & 4 & - & 0 & 0.35 & 5 & - & 0 & 0.45 & 5 & - \\
\hline$\varrho_{11}$ & 0.21 & 0.20 & 0.19 & 0.20 & 0.23 & 0.24 & 0.22 & 0.22 & 0.29 & 0.28 & 0.26 & 0.26 \\
\hline$\varrho_{22}$ & 0.21 & 0.20 & 0.20 & 0.21 & 0.24 & 0.24 & 0.23 & 0.23 & 0.29 & 0.28 & 0.26 & 0.26 \\
\hline$\varrho_{33}$ & 0.22 & 0.21 & 0.21 & 0.21 & 0.25 & 0.25 & 0.24 & 0.23 & 0.30 & 0.30 & 0.29 & 0.28 \\
\hline
\end{tabular}
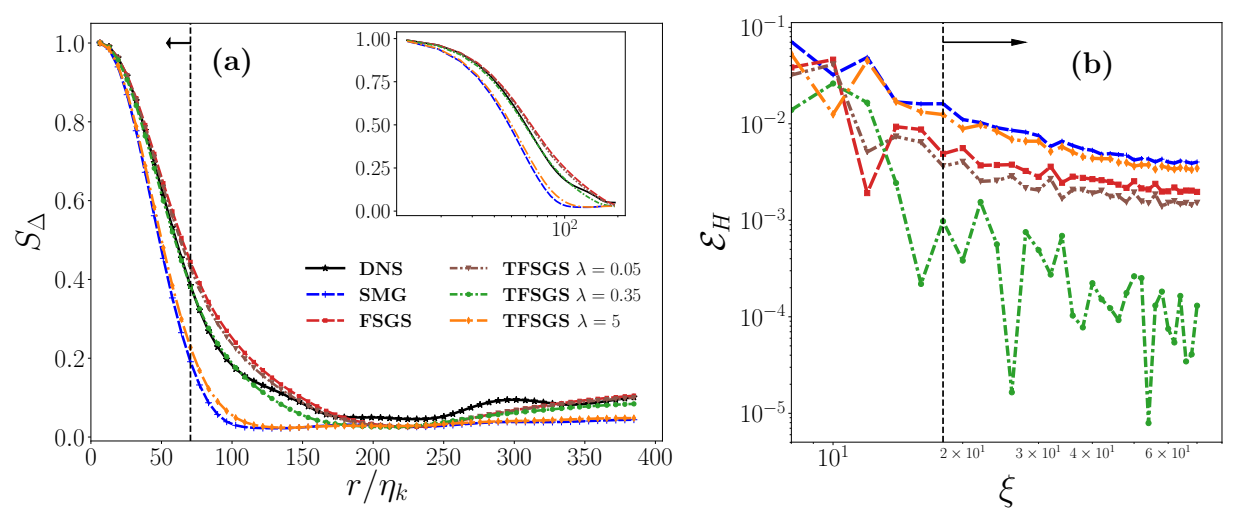

Figure 2: (a) Comparing results of the normalized two-point stress-strain rate correlation functions $\left(S_{\Delta}\right)$ and (b) error analysis of the longitudinal dissipation spectrum $\left(\mathcal{E}_{H}\right)$ for fractional SGS and Smagorinsky models, where $\mathcal{L}_{\delta}=8$ and $\xi$ represents the radius of Fourier wave numbers. In both plots, the arrows point to the dissipation range.

LES models at high wave numbers, we define

$$
\mathcal{E}_{H}=\frac{\left|[\hat{\mathcal{D}}(\xi)]^{*}-[\hat{\mathcal{D}}(\xi)]^{D}\right|}{\left|[\hat{\mathcal{D}}(\xi)]^{D}\right|},
$$

where $|\cdot|$ represents norm of the vector. Figure 2 (a) displays $S_{\Delta}$ versus the spatial shift, $r$, for a logarithmic sequence of $\lambda$ spanning three orders of magnitude in the TFSGS model, where $\mathcal{L}_{\delta}=8$. As stated earlier, the proposed model can take a journey from the FSGS to the SMG models by tuning $\lambda$. Evidently, the true quantities of $S_{\Delta}$, colored by black, are well-predicted by the proposed model with $\lambda^{o p t}=0.45$, where $\alpha^{o p t}=0.58$ is fixed. Figure 2 (b) confirms our findings quantitatively in a plot of $\mathcal{E}_{H}$ versus radius of wave numbers, $\xi$, with log-scale axes. In fact, this plot implies accuracy of the TFSGS model in capturing the two-point structure function at the dissipation range, pointed by an arrow.

Employing the same analysis for $\mathcal{L}_{\delta}=4,12$, we infer the optimal behavior of the TFSGS model, evaluated for a logarithmic range of $\lambda$ with a fixed $\alpha^{o p t}$, in Figure 3 . The inset plots show $\mathcal{E}_{H}$ versus $\xi$ using log-scale on both axes to magnify the dissipation range at high wave numbers. Interestingly, at $\mathcal{L}_{\delta}=4$ the FSGS model is dissipative enough to outperform the tempered model in capturing the true $S_{\Delta}$ in Figure 3 (a). With all this in mind, these results certify the importance 

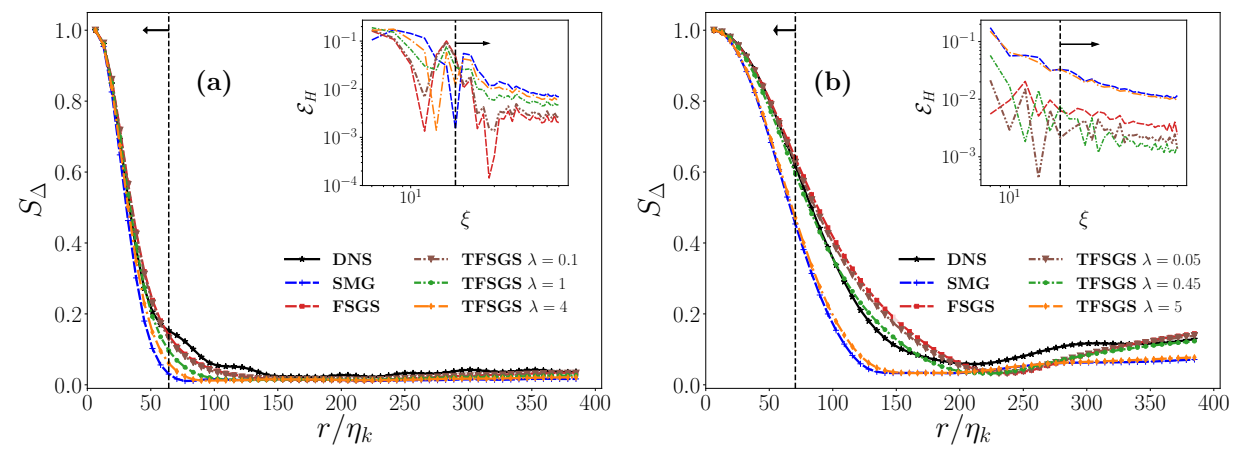

Figure 3: Comparing results of the normalized two-point stress-strain rate correlation functions for (a) $\mathcal{L}_{\delta}=4$ and (b) $\mathcal{L}_{\delta}=12$. The inset plots illustrate the scaled error of longitudinal dissipation spectrum $\left(\mathcal{E}_{H}\right)$ versus the radius of Fourier wave numbers $(\xi)$. The arrows point to the dissipation range.

Table 3: Optimized parameters associated with the TFSGS model in terms of Algorithm 1 for differing filter widths.

$\frac{\mathcal{L}_{\delta}=4}{\frac{\alpha}{0.76} \frac{\lambda}{\simeq 0} \frac{\mathrm{c}_{\alpha, \lambda}}{2.08}} \frac{\mathcal{L}_{\delta}=8}{\frac{\alpha}{0.58} \frac{\lambda}{0.35} \frac{\mathrm{c}_{\alpha, \lambda}}{0.88}} \frac{\frac{\alpha}{0.51} \frac{\lambda}{0.45} \frac{\mathrm{c}_{\alpha, \lambda}}{0.048}}{n}$

of tempering in correct regeneration of two-point correlation functions particularly at larger filter widths $\left(\mathcal{L}_{\delta}=8,12\right)$. Moreover, the SMG model, resembling the TFSGS with $\lambda \sim 5$, exhibits a relatively steeper slope at the dissipation range, which is rooted in the diffusive form of its operator. In this context, tempering plays a crucial role in characterizing dissipation structures by comparing the widening gaps between the asymptotic cases $(\lambda=0.01$ and 5) in Figure 3 (b). This brings up the TFSGS model as a superior physics based model in comparison with its counterparts, i.e., the SMG and the FSGS models.

Given the values of $\alpha^{o p t}$ and $\lambda^{o p t}$, we proceed lastly with quantifying $\mathrm{c}_{\alpha, \lambda}$ as prescribed in Algorithm 1. Under statistically stationary circumstances of the flow field, $\mathrm{c}_{\alpha, \lambda}$ remains fairly unchanged for each $\mathcal{L}_{\delta}$ of interest, as reported in Table 3 . It should be noted that $\mathrm{c}_{\alpha, \lambda}$ is only part of the fractional coefficient, described in (4.4), in order to scale up the model in a constant $R e_{\lambda}$ and $\mathcal{L}_{\delta}$.

\subsection{Interpretation of two-point structure functions}

The third-order structure functions, arising from the $\mathrm{KH}$ equations, provide insights about the statistics of unresolved scales and their strong interactions with large scale motions. As discussed previously in section $4, G_{\Delta}=\frac{G_{L L L}}{\epsilon \mathcal{L}}$, representing the scaled two-point velocity-stress correlation function, is introduced as a sufficient condition for precise regeneration of third-order structure functions and an a priori consistency in LES modeling. Following the derivation of the longitudinal Taylor maicroscale in (Pope 2001, chapter 6), $D_{L L}(r)$ seems to be directly connected to the first-order derivative of $G_{L L L}(r)$ at the dissipation range. This offers the capability of the optimum edition of TFSGS model in capturing $G_{\Delta}$ and thereby fulfilling the essential conditions in (4.2). 

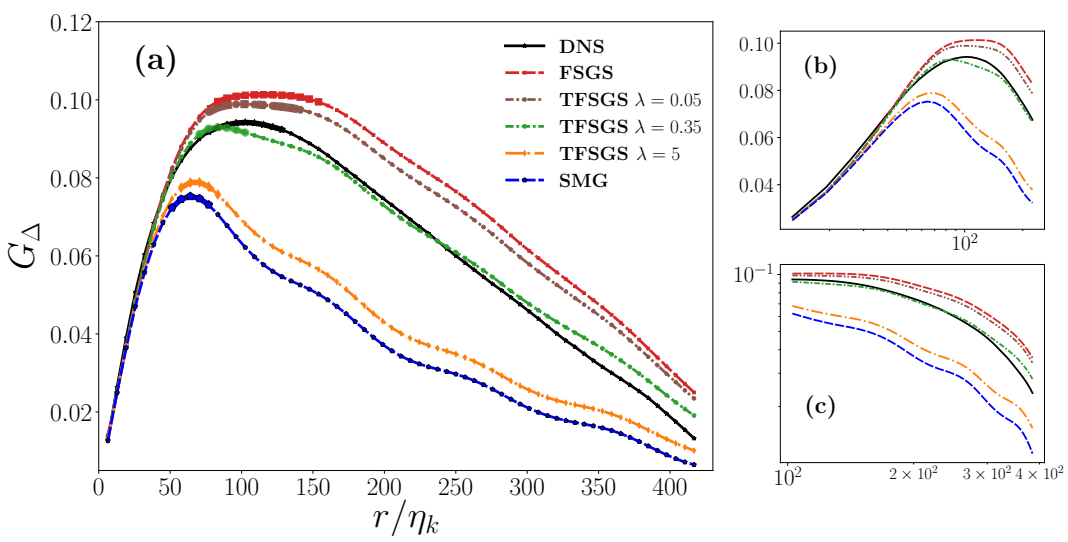

Figure 4: Two-point velocity-stress correlation function in a stationary HIT flow for $\mathcal{L}_{\delta}=8$ using box filtering. The segment of balance region (BR) has been thickened up in (a) for all the graphs. The dissipation and the inertial ranges have been enlarged in plots (b) with semi-logarithmic scale on the $\mathrm{x}$-axis and (c) with logarithmic scale on the both axes, respectively.
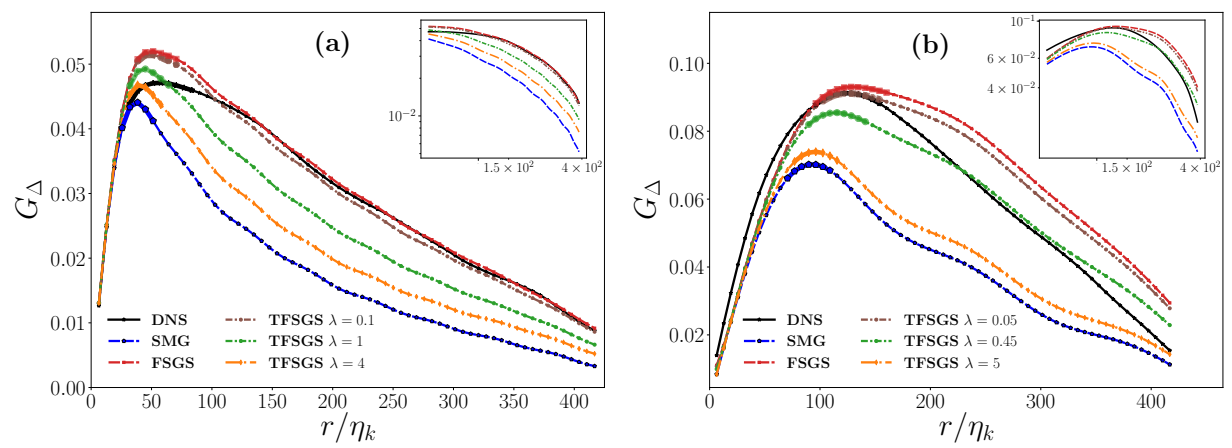

Figure 5: Two-point velocity-stress correlation function in a stationary HIT flow for (a) $\mathcal{L}_{\delta}=4$ and (b) $\mathcal{L}_{\delta}=12$ using box filtering. The inertial range has been enlarged in the inset plots with logarithmic scale on the both axes.

In the first stage of the statistical analysis, we perform a comprehensive study on $G_{\Delta}$ in Figure 4 (a) for $\mathcal{L}_{\delta}=8$, in which the dissipation and inertial ranges are magnified in Figure 4 (b) with semi-logarithmic scale on the x-axis and Figure 4 (c) with logarithmic scale on both axes, respectively. The balance regions (BR), including extremum points, are thickened up in all the graphs in Figure 4 (a). BR also indicates the transitional zone between dissipation and inertial ranges. Aligned with the right side of the (4.2), the trend of $G_{\Delta}$ at small-scale interactions appear to be a linear function of spatial displacement, $r$, suggested by (Meneveau 1994, Figure 2). The results in Figure 4 (a) and more accurately in Figure 4 (b) offer that the optimum TFSGS model well-predict the true DNS quantities at the left side of BR, not only the slope of $G_{\Delta}$ but also the maximum of $G_{\Delta}$ occurring at a relatively close $r$. This spotlights the importance of step three of Algorithm 1 in tuning slope of $G_{\Delta}$ at the dissipation range and effective role of the tempering parameter in fitting the BR, associated with the filtered DNS data. In practice, increasing $\lambda$ pushes BR toward the left side to preserve the increasing linear correlation as a notion of more dissipative behavior. These findings are endorsed qualitatively for the other filter widths in Figure 5 , considering $\lambda^{o p t}$ in Table 3. 

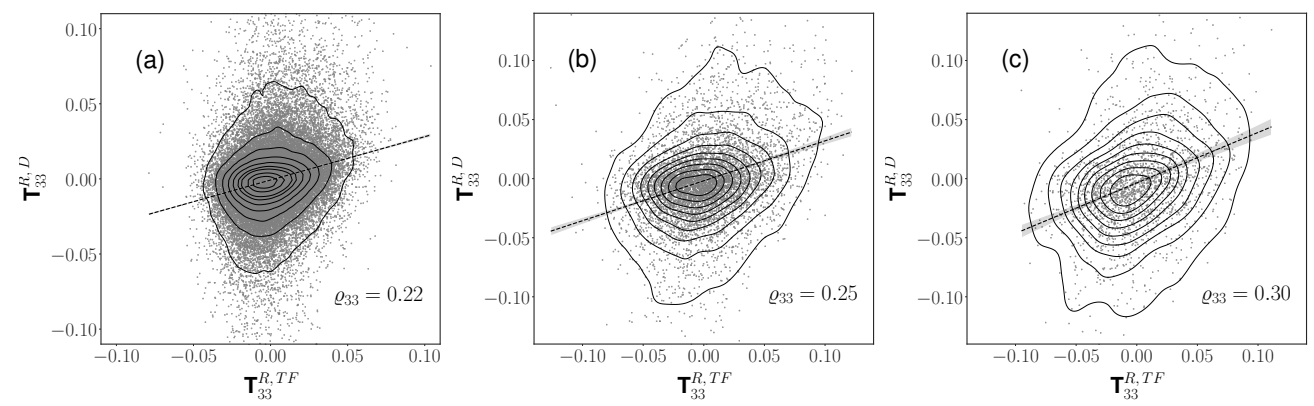

Figure 6: Scatter plots of the SGS stresses obtained by the filtered DNS data $\left(T_{33}^{R, D}\right.$ ) versus the modeled stresses $\left(T_{33}^{R, T F}\right)$ using optimized parameters in Table 3 for (a) $\mathcal{L}_{\delta}=4$, (b) $\mathcal{L}_{\delta}=8$, and (c) $\mathcal{L}_{\delta}=12$.

In analysis of $G_{\Delta}$ at the inertial range, the graph, associated with $\lambda^{o p t}$, shows a favorable match with true points, colored by black, in Figures 4 and 5. For the purpose of clarity, the inertial ranges are magnified in log-log scale plots in Figure 4 (c) for $\mathcal{L}_{\delta}=8$ and the inset plots in Figure 5 for $\mathcal{L}_{\delta}=4,12$, respectively. Motivated by these results, tempered fractional modeling seems to be faithful in fitting structures at the dissipation and the inertial ranges and also estimating the correct value of $r$, associated with the extremum points. Inevitably, enlarging $\mathcal{L}_{\delta}$ accounts for inaccuracies in fitting the tail of graphs as observed in 5 (b). Notwithstanding, the mid-range interactions are acceptably predicted by the optimized TFSGS model.

With an overview of the present results, the TFSGS model stands out as a structure based approach, which reasonably covers the gap between the FSGS and the SMG models. In $\mathcal{L}_{\delta}=4$, $\lambda^{o p t}$ is found to be very close to zero, which renders tempering nonessential in capturing twopoint structures. As we increase $\mathcal{L}_{\delta}$, this gap starts widening up and tempering mechanism acts more dynamically in finding the true BR and fitting the dissipation structures. This argument confirms that the tempered fractional approach displays a great potential for parameterizing structure function especially at larger filter widths while retaining fairly acceptable accuracy.

\subsection{PDF of SGS stresses}

Within the proposed statistical framework, the last step in Algorithm 1 focuses on the PDFs of filtered DNS data. The key idea is to assess the performance of models and verify if the proposed model maintains the true statistics. In this context, we present the scatter plots of $T_{i i}^{R, D}$ against $T_{i i}^{R, T F}$ in Figure 6 for three given filter widths and $i=3$. We should note that the present results are confined to $i=3$ due to the similarities in other directions. The slope in each plot is indicated by the corresponding correlation coefficients in Table 2 . The most noticeable specific about these results is that the data points are bounded within a same order of magnitude on both axes. As a matter of fact, we achieve a roughly unit regression coefficient between $T_{i i}^{R, D}$ and $T_{i i}^{R, T F}$, where our optimization strategy targets for correct estimation of the SGS dissipation. This analysis can be extended to the PDF plots in Figure 7. With nearly the same correlation coefficients, the SMG model fails to reproduce the true statistics, while the optimum TFSGS model offers a great match with the true graphs. As pointed out previously, in $\mathcal{L}_{\delta}=4$ the FSGS model represents the equivalent form of the TFSGS with $\alpha^{o p t}=0.76$ and $\lambda^{o p t} \simeq 0$.

From the understanding of energy cascading in turbulent flows, the SGS dissipation, $\epsilon$, is considered as an external parameter in two-point structure equations for describing small-scale motions. In the statistical sense, we compare the PDFs of $\epsilon$, implied by the models, with the true PDFs, obtained by the filtered DNS data for $\mathcal{L}_{\delta}=4,8,12$. As shown in Figure 8, the fractional models accurately predicts the forward scattering, associated with the positive dissipation, $\epsilon^{+}$, 

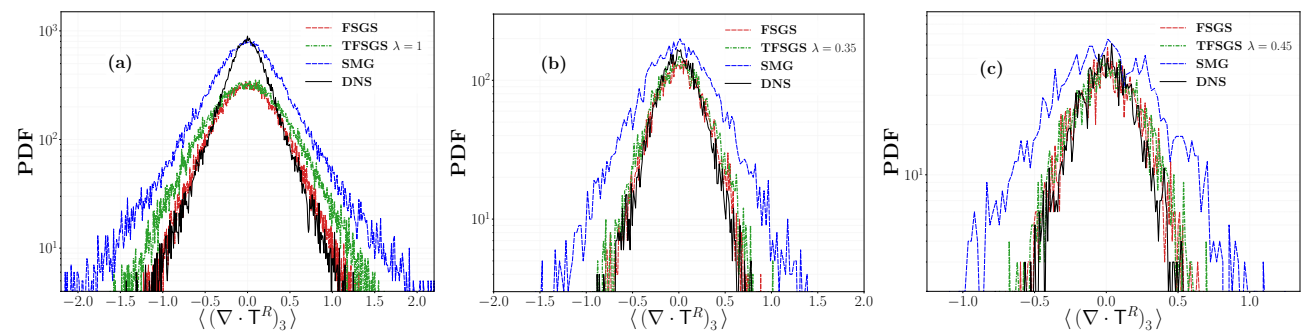

Figure 7: PDF of the ensemble-averaged $(\nabla \cdot T)_{i=3}$ for the optimized (tempered) fractional and the SMG models at (a) $\mathcal{L}_{\delta}=4$, (b) $\mathcal{L}_{\delta}=8$, and (c) $\mathcal{L}_{\delta}=12$.
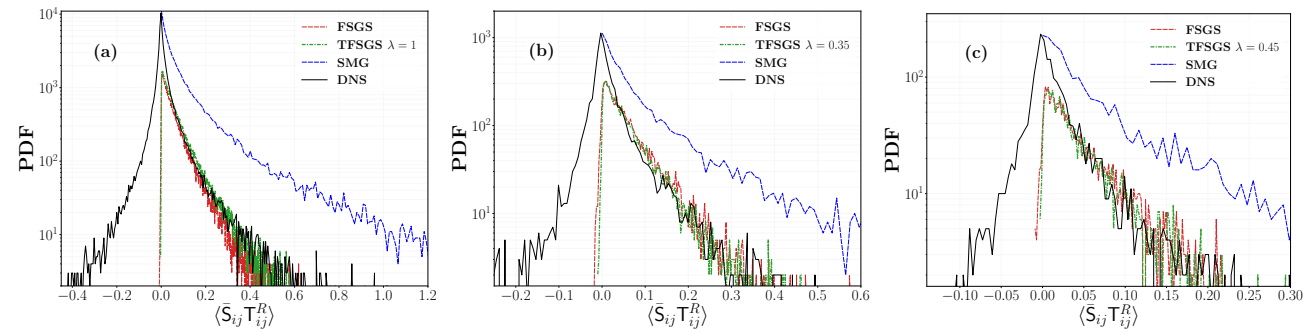

Figure 8: PDF of the ensemble-averaged SGS dissipation for the optimized (tempered) fractional and the SMG models at (a) $\mathcal{L}_{\delta}=4$, (b) $\mathcal{L}_{\delta}=8$, and (c) $\mathcal{L}_{\delta}=12$.

while the SMG model appears to be too dissipative due to its positive eddy viscosity. Furthermore, the TFSGS model presents an under-prediction of the backward scattering by producing a slim amount of negative dissipation, $\epsilon^{-}$. On the side of numerical analysis, this limitation results in preserving numerical stability by minimizing negative dissipation error.

\subsection{A Posteriori Analysis}

With a focus on numerical stability, we extend the statistical a priori assessments to an a posteriori analysis in order to evaluate the performance of proposed models in time. To outline the $a$ posteriori framework, we employ the flow solver, described in section 5.1, on $40^{3}$ and $20^{3}$ grids for the corresponding $\mathcal{L}_{\delta}=4,8$, respectively. The simulations are initiated with an instantaneous flow field, given from the filtered DNS of the stationary HIT flow. This analysis also allows for structural comparisons between the fractional models with the filtered DNS data through the resolved turbulent kinetic energy, $\bar{K}_{t o t}(t)=\left\langle\frac{\bar{V}_{i} \bar{V}_{i}}{2}\right\rangle_{s}$, and the resolved enstrophy, $\overline{\mathcal{E}}(t)=\left\langle\frac{\bar{\omega}_{i} \bar{\omega}_{i}}{2}\right\rangle_{s}$, where $\bar{\omega}_{i}\left(t, x_{i}\right)$ denotes the $i$ th component of the instantaneous filtered vorticity field. It should be noted that in this section $\langle\cdot\rangle_{s}$ denotes the spatial averaging over the entire domain.

Figure 9 displays the decay of kinetic energy in the subgrid-scale level, which verifies the computational stability for the fractional model. In most LES approaches, fidelity in representing spatial structures is essentially compromised to preserve numerical stability by inducing the excessive amount of energy dissipation. Nevertheless, the present results verify our findings in section 4 that the TFSGS model provides stable LES solutions while preserving high-order structure functions in the a priori tests. Comparing the results in Figure 9, the fractional model also exhibits an acceptable performance in predicting the time-evolution of kinetic energy after one or two eddy turn-over times, $\tau_{\mathscr{L}}$, for both resolutions.

Given the analysis in section 4, third-order structure functions are inherently connected with the third-order moments of filtered velocity derivatives and thereby the resolved enstrophy balance as 

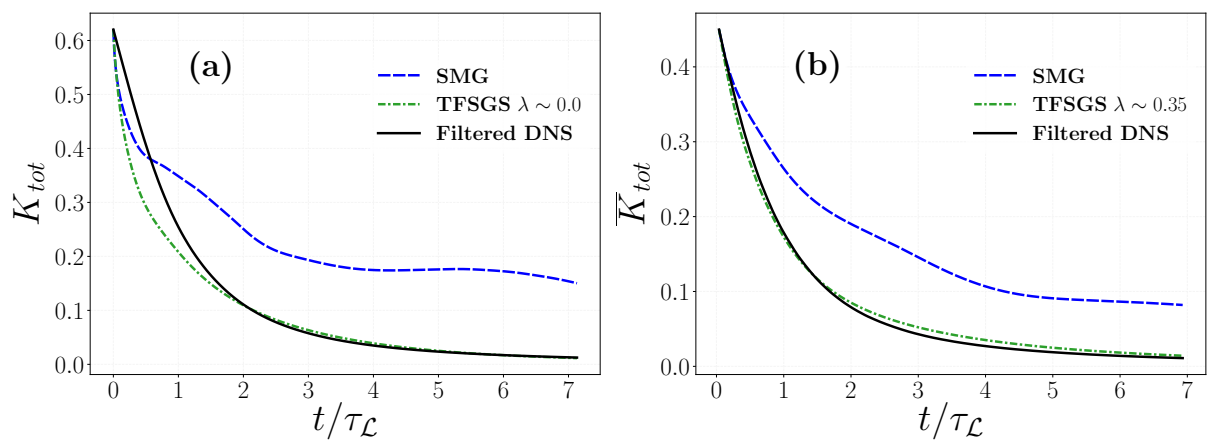

Figure 9: Decay of the resolved turbulent kinetic energy, $\bar{K}_{t o t}$, for the optimized TFSGS and the SMG models with (a) $40^{3}$ and (b) $20^{3}$ grid points.
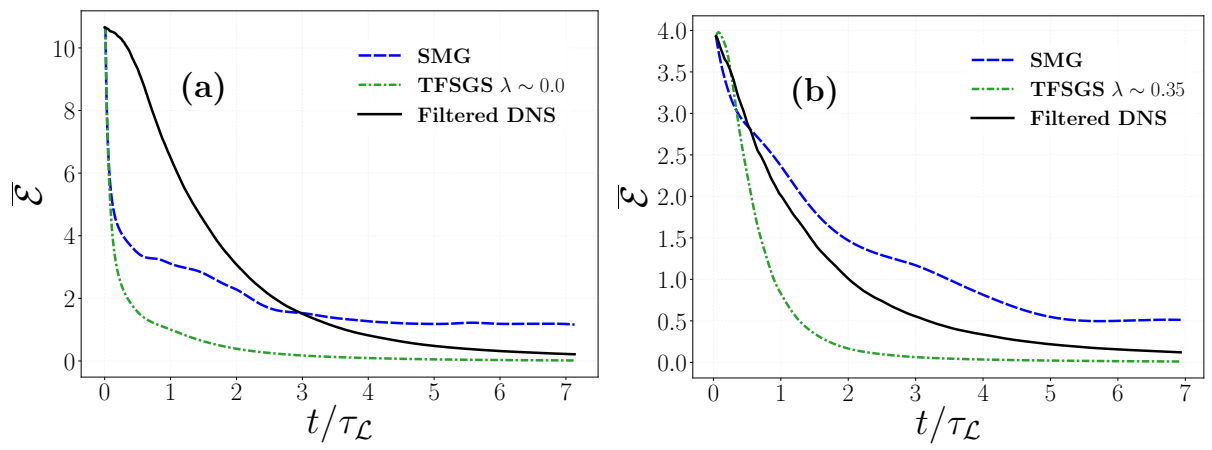

Figure 10: Time-evolution of the resolved enstrophy, $\overline{\mathcal{E}}$, for the optimized TFSGS and the SMG models with (a) $40^{3}$ and (b) $20^{3}$ grid points.

described in (Meneveau 1994). Inspired by that, we also study the time evolution of $\overline{\mathcal{E}}$ in Figure 10 , which evidently confirms numerical stability of the fractional LES solutions in both cases.

\subsection{Merits, challenges, and future works}

On the basis of a priori and a posteriori analyses, the present work provides a robust physics based framework for fractional LES modeling of SGS structures. The significance of this approach lies in:

(i) We treat the source of turbulent small-scale motions at the kinetic level, by employing a tempered heavy-tailed distribution in approximating $\overline{f^{e q}}$. This leads us to the tempered fractional operator in the filtered NS equations as a proper choice for modeling a power-law like behavior in the mid-range and a Gaussian tail in real-physics anomalous phenomena.

(ii) The proposed TFSGS model sets the ground for fulfilling essential statistical conditions as a relatively best approximation of an ideal LES model through fractional and tempering parameters. To achieve an optimized edition of the TFSGS model, we devise an optimization strategy, which involves conventional one-point correlation coefficients, two-point structures, and the SGS dissipation.

(iii) The optimized TFSGS model presents reasonably accurate predictions of two-point structure functions for a range of filter widths while maintaining the expected correlations between the modeled and true SGS stresses. 
(iv) The corresponding fractional LES solutions present a stable prediction of energy and enstrophy decays in the a posteriori analysis.

Despite the theorertical and statistical achievements, we believe that as a viable and promising direction toward nonlocal modelings, this approach can be upgraded to attain better accuracy and involve higher-order statistical properties of turbulent flows. On the theoretical side, the current framework deserves a careful mathematical attention to be extended to anisotropic and inhomogeneous flows employing proper forms of distributions at the kinetic level. Moreover, further works should be undertaken to generalize the fractional model to a data-driven representation of spatial and temporal structures in more complex turbulent regimes.

\section{Conclusions and Remarks}

Inspired by nonlocality, embedded in interactions between large and small scale motions, we developed a tempered fractional SGS model for LES of HIT flows. We began with modeling of turbulent effects at the kinetic level by closing the collision term in the filtered BT equation. To approximate multi-exponential behaviors of the filtered equilibrium distribution in the collision operator, we employed a tempered Lévy-stable distribution function, which presents a power-law at a moderate range and then converges to an exponential decay. By ensemble-averaging of the approximated Boltzmann transport, we derived the LES equations, in which the divergence of SGS stresses emerged as a summation of tempered fractional Laplacian, $(\Delta+\lambda)^{\alpha}(\cdot)$, where $\alpha \in(0,1), \alpha \neq \frac{1}{2}$, and $\lambda>0$. Interestingly, the FSGS is found to be a particular form of the TFSGS model when $\lambda$ approaches 0. Moreover, we formulated the SGS stresses straightforwardly in terms of a combination of integer and fractional operators, which gives the advantage of being feasible and quite easy to implement in the Fourier space. The corollary on frame invariant property of the FSGS model were also extended to the current model, showing its physical and mathematical consistency.

In a statistical framework, we constructed a structure based algorithm for optimizing the fractional models, which involved the closed essential conditions for a weaker sense of an ideal LES model. Following the optimization strategy, we inferred the optimum tempering parameter through a comparative study of two-point strain-stress correlation functions while the fractional exponent was fixed for maintaining reasonable values of correlation coefficients. Next, we quantified the fractional coefficient using SGS dissipation as a crucial factor in identifying high-order structures. The more profound analysis of dissipation structure functions emphasized on the central role of $\lambda$ in spanning the widening gap between the FSGS and the SMG models, especially at larger $\mathcal{L}_{\delta}$, when $\alpha^{o p t}$ decreases. Regarding the KH equation, the optimum TFSGS model presented a great match with the true values of two-point velocity-stress correlation functions, which ensures the accurate prediction of third-order structure functions.

The success of tempering mechanism in capturing structure correlation functions, particularly at larger $\mathcal{L}_{\delta}$, originated from the capabilities of our choice in fitting semi-heavy-tailed behavior of the filtered equilibrium distribution at the kinetic level. The inspection of statistical results also supported accuracy of the fractional model in keeping unit regression and capturing the corresponding PDF tails. As a notion of numerical stability, we demonstrated that the optimized TFSGS model well-predicted the true forward scattering in a statistical sense without generating any significant negative dissipation.

Lastly, the TFSGS model underwent the ultimate a posteriori analysis, which verified numerically stable performance of the fractional model through tracking turbulent kinetic energy and enstrophy. With the emphasis on remarkable potentials and merits of the present work, we believe that this approach can be extended to more complex turbulent flows by employing a variety of rigorous fractional operators, derived from the statistical structures. 


\section{Acknowledgment}

This work was financially supported by the MURI/ARO grant (W911NF-15-1-0562), the ARO Young Investigator Program (YIP) award (W911NF-19-1-0444), and partially by the National Science Foundation award (DMS-1923201). The high-performance computing resources and services were provided by the Institute for Cyber-Enabled Research (ICER) at Michigan State University.

\section{Appendix A.}

As noted in (Deng et al. 2018; Di Nezza et al. 2012), the tempered fractional Laplacian operator can be represented in various equivalent forms, i.e.,

$$
\begin{aligned}
(\Delta+\lambda)^{\alpha} u(\boldsymbol{x}) & =-C_{d, \alpha} \text { P.V. } \int_{\mathbb{R}^{d}} \frac{u(\boldsymbol{x})-u(\mathfrak{s})}{e^{\lambda|\boldsymbol{x}-\mathfrak{s}|}|\boldsymbol{x}-\mathfrak{s}|^{2 \alpha+d}} d \mathfrak{s} \\
& =\frac{C_{d, \alpha}}{2} \mathrm{P} . \mathrm{V} . \int_{\mathbb{R}^{d}} \frac{u(\boldsymbol{x}+\mathfrak{s})+u(\boldsymbol{x}-\mathfrak{s})-2 u(\boldsymbol{x})}{e^{\lambda \mathfrak{s} \mathfrak{s}^{2 \alpha+d}}} d \mathfrak{s},
\end{aligned}
$$

where $\mathfrak{s}=|\mathfrak{s}|, \alpha \in\left(0, \frac{1}{2}\right) \cup\left(\frac{1}{2}, 1\right)$, and $\lambda>0$. By performing the Fourier transform of (A 1), we get

$$
\begin{aligned}
\mathcal{F}\left[(\Delta+\lambda)^{\alpha} u(\boldsymbol{x})\right](\boldsymbol{\xi}) & =\frac{C_{d, \alpha}}{2} \int_{\mathbb{R}^{d}} \frac{e^{\xi \cdot \mathfrak{s}}+e^{-\xi \cdot \mathfrak{s}}-2}{e^{\lambda \mathfrak{s}} s^{2 \alpha+d}} d \mathfrak{s} \\
& =-C_{d, \alpha} \int_{\mathbb{R}^{d}} \frac{1-\cos (\boldsymbol{\xi} \cdot \mathfrak{s})}{e^{\lambda \mathfrak{s}} \mathfrak{s}^{n+2 \alpha}} d \mathfrak{s} \mathcal{F}[u(\boldsymbol{x})](\boldsymbol{\xi}),
\end{aligned}
$$

in which $\boldsymbol{\xi}$ denotes the Fourier numbers. For the sake of simplicity, we define

$$
I(\boldsymbol{\xi})=-\int_{\mathbb{R}^{d}} \frac{1-\cos (\boldsymbol{\xi} \cdot \mathfrak{s})}{e^{\lambda \mathfrak{s}} \mathfrak{s}^{n+2 \alpha}} d \mathfrak{s},
$$

which appears to be rotationally invariant. Moreover, we introduce $\xi=|\boldsymbol{\xi}|$ and $\mathfrak{s}_{\theta}=\mathfrak{s} \cos (\theta)$. Without loss of generality, $\theta$ can be chosen such that $\mathfrak{s} \cos (\theta)$ is aligned with the first primary direction. Therefore, $I(\xi)$ can be re-expressed by

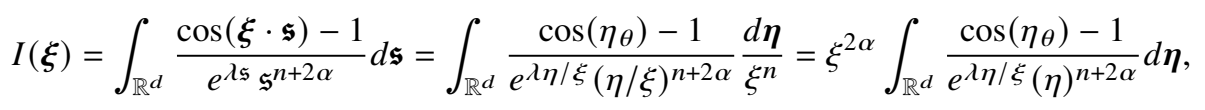

where $\boldsymbol{\eta}=\boldsymbol{\xi} \mathfrak{s}, \eta=|\boldsymbol{\eta}|$, and $\eta_{\theta}=\xi_{\mathfrak{s}_{\theta}}$. Due to the invariant properties of $I(\boldsymbol{\xi})$, we proceed the derivations with transforming (A 3) into the corresponding spherical coordinate, $\left(\mathrm{r}, \phi_{1}, \cdots, \phi_{d-1}\right)$.

In terms of the transformation, we let $\eta=|\boldsymbol{\eta}|=\mathrm{r}$ and $\eta_{\theta}=\eta \cos (\theta)=\mathrm{r} \cos \left(\phi_{1}\right)$. Then, in a general case for $d>1, d \boldsymbol{\eta}$ follows

$$
d \boldsymbol{\eta}=\mathcal{J}\left(\mathrm{r}, \phi_{1}, \cdots, \phi_{d-1}\right) d \mathrm{r} d \phi_{1} \cdots d \phi_{d-1},
$$

where

$$
\mathcal{J}\left(\mathrm{r}, \phi_{1}, \cdots, \phi_{d-1}\right)=\left|\operatorname{det} \frac{\partial x_{i}}{\partial\left(\mathrm{r} \phi_{j}\right)}\right|=\mathrm{r}^{d-1} \sin ^{d-2}\left(\phi_{1}\right) \sin ^{d-3}\left(\phi_{2}\right) \cdots \sin \left(\phi_{d-2}\right)
$$

for $i=1, \cdots, d$ and $j=1, \cdots, d-1$ (see Henderson \& Taimina 2000). Therefore, we find the general form of $I(\xi)$ as

$$
I(\xi)=\xi^{2 \alpha} \bar{c} \int_{0}^{\infty} \int_{0}^{2 \pi} \frac{\cos \left(\mathrm{r} \cos \left(\phi_{1}\right)\right)-1}{e^{\lambda \mathrm{r} / \xi}(\mathrm{r})^{d+2 \alpha}} \mathrm{r}^{d-1} \sin ^{d-2}\left(\phi_{1}\right) d \phi_{1} d \mathrm{r},
$$


where $\bar{c}=\int_{0}^{\pi} \sin ^{d-3}\left(\phi_{2}\right) d \phi_{2} \cdots \int_{0}^{\pi} \sin \left(\phi_{d-1}\right) d \phi_{d-1}=\frac{2 \pi^{(d-1) / 2}}{\Gamma\left(\frac{d-1}{2}\right)}$. It is shown by Deng et al. (2018) that

$$
\begin{aligned}
I(\boldsymbol{\xi}) & =\bar{c} \xi^{2 \alpha} \int_{0}^{\infty} \frac{e^{-\lambda \mathrm{r} / \xi}}{\mathrm{r}^{\beta+1}} \int_{0}^{2 \pi}\left[\cos \left(\mathrm{r} \cos \left(\phi_{1}\right)\right)-1\right] \sin ^{d-2}\left(\phi_{1}\right) d \phi_{1} d \mathrm{r} \\
& =\frac{\bar{c} \Gamma(-2 \alpha) \pi^{1 / 2} \Gamma\left(\frac{d-1}{2}\right)}{\Gamma\left(\frac{d}{2}\right)}\left[\lambda^{2}-\left(\lambda^{2}+\xi^{2}\right)^{\alpha}{ }_{2} F_{1}\left(-\alpha, \frac{d+2 \alpha-1}{2} ; \frac{d}{2} ; \frac{\xi^{2}}{\xi^{2}+\lambda^{2}}\right)\right],
\end{aligned}
$$

where ${ }_{2} F_{1}$ denotes a Gaussian hypergeometic function. Therefore,

$\mathcal{F}\left[(\Delta+\lambda)^{\alpha} u(\boldsymbol{x})\right](\boldsymbol{\xi})=\mathfrak{C}_{d, \alpha} \times\left[\lambda^{2}-\left(\lambda^{2}+\xi^{2}\right)^{\alpha}{ }_{2} F_{1}\left(-\alpha, \frac{d+2 \alpha-1}{2} ; \frac{d}{2} ; \frac{\xi^{2}}{\xi^{2}+\lambda^{2}}\right)\right]$,

where $\mathfrak{C}_{d, \alpha}=C_{d, \alpha} \bar{c} \Gamma(-2 \alpha) \frac{\pi^{1 / 2} \Gamma\left(\frac{d-1}{2}\right)}{\Gamma(d / 2)}=\frac{1}{{ }_{2} F_{1}\left(-\alpha, \frac{d+2 \alpha-1}{2} ; \frac{d}{2} ; 1\right)}$.

\section{Appendix B.}

As we discussed in subsection 3.3, the SGS stresses are described by

$$
T_{i j}^{R}=\frac{\rho \mathrm{c}_{\beta, \lambda}}{U^{3}} \int_{0}^{\infty} \int_{\mathbb{R}^{3}}\left(u_{i}-\bar{V}_{i}\right)\left(u_{j}-\bar{V}_{j}\right)\left(F^{\beta, \lambda}\left(\bar{\Delta}_{s}\right)-F^{\beta, \lambda}(\bar{\Delta})\right) e^{-s} d \boldsymbol{u} d s
$$

where $F^{\beta, \lambda}(\bar{\Delta})$ represents a tempered Lévy $\beta$-stable distribution. Let consider $\beta=-\alpha-\frac{3}{2}$ for $\alpha \in\left(0, \frac{1}{2}\right) \cup\left(\frac{1}{2}, 1\right)$. Regarding the equivalent Pareto-like behavior of Lévy distributions (Weron 2001) at $\bar{\Delta}>1$, we decompose the domain of kinetic momentum such that $\mathbb{R}^{3}=\mathcal{I}_{\epsilon} \cup\left(\mathbb{R}^{3} \backslash \mathcal{I}_{\epsilon}\right)$, where $\mathcal{I}_{\epsilon}=\left\{u \in \mathbb{R}^{d}\right.$ s.t. $\left.|\bar{\Delta}|<\epsilon\right\}$ and $\epsilon \ll 1$. This allows for the following approximation as

$$
F^{\alpha, \lambda}(\bar{\Delta}) \simeq C_{\alpha} \begin{cases}0, & \boldsymbol{u} \in \mathcal{I}_{\epsilon} \\ e^{-\lambda \bar{\Delta}^{\frac{1}{2}}} \bar{\Delta}^{-\left(\alpha+\frac{3}{2}\right)}, & \boldsymbol{u} \in \mathbb{R}^{3} \backslash \mathcal{I}_{\epsilon}\end{cases}
$$

where $C_{\alpha}=\frac{-\Gamma\left(\frac{3}{2}\right)}{2 \pi^{\frac{3}{2}} \Gamma(-2 \alpha)} \frac{1}{{ }_{2} F_{1}\left(-\alpha, 1+\alpha ; \frac{3}{2} ; 1\right)}$. It is worth mentioning that $F^{\alpha, \lambda}(\bar{\Delta})$ reduces exponentially in a close proximity of $\bar{\Delta}=0$. With all this in mind, the approximated function of $F^{\alpha, \lambda}(\bar{\Delta})$ in (B 2) can properly capture the heavy-tailed behavior of the filtered collision term. Evidently, by replacing $e^{-\lambda \bar{\Delta}_{s}^{\frac{1}{2}}}$ with $e^{-\lambda \bar{\Delta}^{\frac{1}{2}}}$ for $\bar{\Delta}>1$, we arrive at the following expression

$$
F^{\alpha, \lambda}\left(\bar{\Delta}_{s}\right)-F^{\alpha, \lambda}(\bar{\Delta})=C_{\alpha}\left(\frac{e^{-\lambda \bar{\Delta}_{s}^{\frac{1}{2}}}}{\bar{\Delta}_{s}^{\left(\alpha+\frac{3}{2}\right)}}-\frac{e^{-\lambda \bar{\Delta}^{\frac{1}{2}}}}{\bar{\Delta}^{\left(\alpha+\frac{3}{2}\right)}}\right) \simeq C_{\alpha} e^{-\lambda \bar{\Delta}^{\frac{1}{2}}}\left(\frac{1}{\bar{\Delta}_{S}^{\left(\alpha+\frac{3}{2}\right)}}-\frac{1}{\bar{\Delta}^{\left(\alpha+\frac{3}{2}\right)}}\right),
$$

and accordingly,

$$
T_{i j}^{R}=\frac{\rho \mathrm{c}_{\alpha, \lambda}}{U^{3}} C_{\alpha} \int_{0}^{\infty} \int_{\mathbb{R}^{3} \backslash I_{\Delta}}\left(u_{i}-\bar{V}_{i}\right)\left(u_{j}-\bar{V}_{j}\right) e^{-\lambda \bar{\Delta}^{\frac{1}{2}}}\left(\frac{1}{\bar{\Delta}_{s}^{\left(\alpha+\frac{3}{2}\right)}}-\frac{1}{\bar{\Delta}^{\left(\alpha+\frac{3}{2}\right)}}\right) e^{-s} d \boldsymbol{u} d s,(\mathrm{~B}
$$

where $\mathrm{c}_{\alpha, \lambda}$ is a real-valued constant. As a continuous differentiable function for $\bar{\Delta}>1$, we proceed with the Taylor expansion of $\bar{\Delta}_{S}^{-\left(\alpha+\frac{3}{2}\right)}$ according to

$$
\bar{\Delta}_{S}^{-\left(\alpha+\frac{3}{2}\right)}-\bar{\Delta}^{-\left(\alpha+\frac{3}{2}\right)} \approx \frac{\partial \bar{\Delta}^{-\left(\alpha+\frac{3}{2}\right)}}{\partial \bar{\Delta}}\left(\bar{\Delta}_{s}-\bar{\Delta}\right)=-\left(\alpha+\frac{3}{2}\right) C_{\alpha} \frac{\left(\bar{\Delta}_{s}-\bar{\Delta}\right)}{\bar{\Delta}^{\alpha+5 / 2}} .
$$


In terms of the assumptions in remark 1, we use the same argument, presented by (Samiee et al. 2020a, Appendix), on approximating $\bar{\Delta}_{s}-\bar{\Delta}$ for $\bar{\Delta} \gg 1$, which allows for $u_{i}-\bar{V}_{i} \approx u_{i}$ and thus

$$
\bar{\Delta}_{s}-\bar{\Delta} \approx 2 \sum_{k=1}^{3} \frac{u_{k}\left(\bar{V}_{k}\left(\boldsymbol{x}^{\prime}\right)-\bar{V}_{k}(\boldsymbol{x})\right)}{U^{2}}
$$

Reminding the definition of $\boldsymbol{u}=\frac{\boldsymbol{x}-\boldsymbol{x}^{\prime}}{\boldsymbol{s} \boldsymbol{\tau}}$ from section 3.2, we plug (B 4) into (B 3) and obtain

$$
\begin{aligned}
& T_{i j}^{R}=(2 \alpha+3)\left(\rho \mathrm{c}_{\alpha, \lambda} C_{\alpha} \tau^{2 \alpha-1} U^{2 \alpha}\right) \times \\
& \int_{0}^{\infty} \frac{e^{-s}}{s^{1-2 \alpha}} \int_{\mathbb{R}^{3} \backslash I_{\Delta}}\left(x_{i}-x_{i}^{\prime}\right)\left(x_{j}-x_{j}^{\prime}\right) \frac{\left(\boldsymbol{x}-\boldsymbol{x}^{\prime}\right) \cdot\left(\overline{\boldsymbol{V}}(\boldsymbol{x})-\overline{\boldsymbol{V}}\left(\boldsymbol{x}^{\prime}\right)\right)}{\left|\boldsymbol{x}-\boldsymbol{x}^{\prime}\right|^{2 \alpha+5} e^{\lambda \frac{\left|\boldsymbol{x}-\boldsymbol{x}^{\prime}\right|}{s \tau U}}} d \boldsymbol{x}^{\prime} d s .
\end{aligned}
$$

In order to evaluate the outer integral in B 5 and find the corresponding coefficient, our approach is to dissociate the temporal element by employing the bionomial series of $e^{\lambda \frac{\left|x-x^{\prime}\right|}{s \tau U}}$ as follows:

$$
\begin{aligned}
e^{-\frac{\lambda\left|\boldsymbol{x}-\boldsymbol{x}^{\prime}\right|}{s \tau U}} & =\left(1-1+e^{-\frac{\lambda\left|\boldsymbol{x}-\boldsymbol{x}^{\prime}\right|}{s \tau U}}\right)^{\frac{1}{s}}=\sum_{k=0}^{\infty}\left(\begin{array}{l}
\frac{1}{s} \\
k
\end{array}\right)\left(e^{\bar{\lambda}\left|\boldsymbol{x}-\boldsymbol{x}^{\prime}\right|}-1\right) \\
& =1+\frac{1}{s}\left(e^{\bar{\lambda}\left|\boldsymbol{x}-\boldsymbol{x}^{\prime}\right|}-1\right)+\frac{\frac{1}{s}\left(\frac{1}{s}-1\right)}{2 !}\left(e^{\bar{\lambda}\left|\boldsymbol{x}-\boldsymbol{x}^{\prime}\right|}-1\right)+\cdots \\
& =\sum_{k=0}^{\infty} W_{k, \infty}(s) e^{-\bar{\lambda}_{k}\left|\boldsymbol{x}-\boldsymbol{x}^{\prime}\right|} \\
& \simeq \sum_{k=0}^{\mathcal{K}} W_{k, \mathcal{K}}(s) e^{-\bar{\lambda}_{k}\left|\boldsymbol{x}-\boldsymbol{x}^{\prime}\right|}
\end{aligned}
$$

where $\bar{\lambda}=\frac{\lambda}{\tau U}$ and $\bar{\lambda}_{k}=\bar{\lambda}$. Under the assumption of $\lambda>0.01$, we can approximate the binomial series with the first two leading terms, which yields $W_{0,1}=1-\frac{1}{s}$ and $W_{1,1}=\frac{1}{s}$ for $\mathcal{K}=1$. Accordingly, by defining $\bar{v}_{\alpha}=(2 \alpha+3)\left(\rho C_{\alpha} \tau^{2 \alpha-1} U^{2 \alpha}\right)$ and

$$
\bar{\phi}_{k}^{\mathcal{K}}(\alpha)=\int_{0}^{\infty} \frac{e^{-s}}{s^{1-2 \alpha}} W_{k, \mathcal{K}}(s) d s,
$$

we obtain the closed form of $T_{i j}^{R}$ as

$$
T_{i j}^{R}=\mathrm{c}_{\alpha, \lambda} \bar{v}_{\alpha} \sum_{k=0}^{\mathcal{K}} \bar{\phi}_{k}^{\mathcal{K}}(\alpha) \int_{\mathbb{R}^{d}-B_{\epsilon}} \underbrace{\left(x_{i}-x_{i}^{\prime}\right)\left(x_{j}-x_{j}^{\prime}\right) \frac{\left(\boldsymbol{x}-\boldsymbol{x}^{\prime}\right) \cdot\left(\overline{\boldsymbol{V}}-\overline{\boldsymbol{V}}^{\prime}\right)}{\left|\boldsymbol{x}-\boldsymbol{x}^{\prime}\right|^{2 \alpha+5} e^{\bar{\lambda}_{k}\left|\boldsymbol{x}-\boldsymbol{x}^{\prime}\right|}}}_{\mathcal{I}_{i j}} d \boldsymbol{x}^{\prime} .
$$

To ensue the proper form of the SGS stresses in the filtered NS equations, we take the derivative 
of $\mathcal{I}_{i j}$ term by term, which yields

$$
\begin{aligned}
\frac{\partial \mathcal{I}_{i j}}{\partial x_{i}}=\int_{\mathbb{R}^{d}-B_{\epsilon}} \sum_{i=1}^{3}\{ & -\left(x_{j}-x_{j}^{\prime}\right) \frac{\left(\boldsymbol{x}-\boldsymbol{x}^{\prime}\right) \cdot\left(\overline{\boldsymbol{V}}-\overline{\boldsymbol{V}}^{\prime}\right)}{\left|\boldsymbol{x}-\boldsymbol{x}^{\prime}\right|^{2 \alpha+5} e^{\bar{\lambda}_{k}\left|\boldsymbol{x}-\boldsymbol{x}^{\prime}\right|}} \\
& -\left(x_{i}-x_{i}^{\prime}\right) \delta_{i j} \frac{\left(\boldsymbol{x}-\boldsymbol{x}^{\prime}\right) \cdot\left(\overline{\boldsymbol{V}}-\overline{\boldsymbol{V}}^{\prime}\right)}{\left|\boldsymbol{x}-\boldsymbol{x}^{\prime}\right|^{2 \alpha+5} e^{\bar{\lambda}_{k}\left|\boldsymbol{x}-\boldsymbol{x}^{\prime}\right|}} \\
& -\frac{\left(x_{j}-x_{j}^{\prime}\right)\left(x_{j}-x_{j}^{\prime}\right)\left(\bar{V}_{i}-\bar{V}_{i}^{\prime}\right)}{\left|\boldsymbol{x}-\boldsymbol{x}^{\prime}\right|^{2 \alpha+5} e^{\bar{\lambda}_{k}\left|\boldsymbol{x}-\boldsymbol{x}^{\prime}\right|}} \\
& -\left(x_{i}-x_{i}^{\prime}\right)\left(x_{j}-x_{j}^{\prime}\right)\left(x_{k}-x_{k}^{\prime}\right) \frac{\partial \bar{V}_{k}}{\partial x_{i}} \frac{e^{-\bar{\lambda}_{k}\left|\boldsymbol{x}-\boldsymbol{x}^{\prime}\right|}}{\left|\boldsymbol{x}-\boldsymbol{x}^{\prime}\right|^{2 \alpha+5}} \\
& (2 \alpha+5)\left(x_{i}-x_{i}^{\prime}\right)^{2}\left(x_{j}-x_{j}^{\prime}\right) \frac{\left(\boldsymbol{x}-\boldsymbol{x}^{\prime}\right) \cdot\left(\overline{\boldsymbol{V}}-\overline{\boldsymbol{V}}^{\prime}\right)}{\left|\boldsymbol{x}-\boldsymbol{x}^{\prime}\right|^{2 \alpha+5} e^{\bar{\lambda}_{k}\left|\boldsymbol{x}-\boldsymbol{x}^{\prime}\right|}} \\
& +\bar{\lambda}_{k}\left(x_{i}-x_{i}^{\prime}\right)\left(x_{j}-x_{j}^{\prime}\right) \frac{\left(\boldsymbol{x}-\boldsymbol{x}^{\prime}\right) \cdot\left(\overline{\boldsymbol{V}}-\overline{\boldsymbol{V}}^{\prime}\right)}{\left.\left|\boldsymbol{x}-\boldsymbol{x}^{\prime}\right|^{2 \alpha+5} e^{\bar{\lambda}_{k}\left|\boldsymbol{x}-\boldsymbol{x}^{\prime}\right|}\right\} d \boldsymbol{x}^{\prime},}
\end{aligned}
$$

which is clearly simplified to

$$
\frac{\partial \mathcal{I}_{i j}}{\partial x_{i}}=\left(\bar{\lambda}_{k}+2 \alpha+5-3-1-1\right) \int_{\mathbb{R}^{d}-B_{\epsilon}}\left(x_{j}-x_{j}^{\prime}\right) \frac{\left(\boldsymbol{x}-\boldsymbol{x}^{\prime}\right) \cdot\left(\overline{\boldsymbol{V}}-\overline{\boldsymbol{V}}^{\prime}\right)}{\left|\boldsymbol{x}-\boldsymbol{x}^{\prime}\right|^{2 \alpha+5} e^{\bar{\lambda}_{k}\left|\boldsymbol{x}-\boldsymbol{x}^{\prime}\right|}} d \boldsymbol{x}^{\prime} .
$$

Following the derivations in (Samiee et al. 2020a; Epps \& Cushman-Roisin 2018), (B 9) can be formulated in the form of a tempered fractional Laplacian by performing the technique of integration-by-parts for (B 9) as $\int A d B=A B-\int B d A$. We consider

$$
A=\left(x_{j}-x_{j}^{\prime}\right)\left(\bar{V}_{k}-\bar{V}_{k}^{\prime}\right) e^{-\bar{\lambda}_{k}\left|\boldsymbol{x}-\boldsymbol{x}^{\prime}\right|}, \quad d B=\frac{\left(x_{k}-x_{k}^{\prime}\right)}{\left|\boldsymbol{x}-\boldsymbol{x}^{\prime}\right|^{2 \alpha+5}} d \boldsymbol{x}^{\prime},
$$

which directly leads to $\left.A B\right|_{u \in \mathbb{R}^{3}} \simeq 0$. Therefore, we get $\int A d B=-\int B d A$, in which

$$
\begin{aligned}
B & =\frac{-1}{(2 \alpha+3)\left|\boldsymbol{x}-\boldsymbol{x}^{\prime}\right|^{2 \alpha+3}}, \\
d A & =\delta_{j k}\left(\bar{V}_{k}-\bar{V}_{k}^{\prime}\right) e^{-\bar{\lambda}_{k}\left|\boldsymbol{x}-\boldsymbol{x}^{\prime}\right|}+\left(x_{j}-x_{j}^{\prime}\right)\left(\frac{\partial \bar{V}_{k}}{\partial x_{k}}\right) e^{-\bar{\lambda}_{k}\left|\boldsymbol{x}-\boldsymbol{x}^{\prime}\right|}-\bar{\lambda}_{k}\left(x_{j}-x_{j}^{\prime}\right)\left(\bar{V}_{k}-\bar{V}_{k}^{\prime}\right) e^{-\bar{\lambda}_{k}\left|\boldsymbol{x}-\boldsymbol{x}^{\prime}\right|} d \boldsymbol{x}^{\prime} .
\end{aligned}
$$

We can make even more simplifications by eliminating the second term of $d A$ due to the incompressibility assumption, i.e., $\partial \bar{V}_{k} / \partial x_{k}=0$. Moreover, by evaluating $\int B d A$ the last term vanishes since it represents an odd function of $\boldsymbol{x}^{\prime}$. Therefore, the ultimate form of the TFSGS model is found to be

$$
\begin{aligned}
\left(\nabla \cdot \boldsymbol{T}^{\mathcal{R}}\right)_{j} & =\mathrm{c}_{\alpha, \lambda} \bar{v}_{\alpha} \sum_{k=0}^{\mathcal{K}} \frac{\left(2 \alpha+\bar{\lambda}_{k}\right)}{(2 \alpha+3)} \bar{\phi}_{k}^{\mathcal{K}}(\alpha) \int_{\mathbb{R}^{d}-B_{\epsilon}} \frac{\left(\bar{V}_{j}-\bar{V}_{j}^{\prime}\right)}{\left|\boldsymbol{x}-\boldsymbol{x}^{\prime}\right|^{2 \alpha+3} e^{\bar{\lambda}_{k}\left|\boldsymbol{x}-\boldsymbol{x}^{\prime}\right|}} d \boldsymbol{x}^{\prime}, \\
& =v_{\alpha} \sum_{k=0}^{\mathcal{K}} \phi_{k}^{\mathcal{K}}\left(\alpha, \bar{\lambda}_{k}\right)\left(\Delta+\bar{\lambda}_{k}\right)^{\alpha} \bar{V}_{j},
\end{aligned}
$$

where $v_{\alpha}=\mathrm{c}_{\alpha, \lambda} \bar{v}_{\alpha}$.

\section{REFERENCES}

Akhavan-Safaei, Ali, Samiee, Mehdi \& Zayernouri, Mohsen 2020a Data-Driven Fractional Subgridscale Modeling for Scalar Turbulence: A Nonlocal LES Approach. arXiv preprint arXiv:2012.14027 
Akhavan-Safaei, Ali, Seyedi, S Hadi \& Zayernouri, Mohsen $2020 b$ Anomalous features in internal cylinder flow instabilities subject to uncertain rotational effects. Physics of Fluids 32 (9), 094107.

Akhavan-Safaei, Ali \& Zayernouri, Mohsen 2020 A Parallel Integrated Computational-Statistical Platform for Turbulent Transport Phenomena. arXiv preprint arXiv:2012.04838 .

Beck, Andrea \& Kurz, Marius 2020 A Perspective on Machine Learning Methods in Turbulence Modelling. arXiv preprint arXiv:2010.12226 .

Bouffanais, Roland 2010 Advances and challenges of applied large-eddy simulation. Computers \& Fluids 39 (5), 735-738.

Briard, Antoine, Gomez, Thomas \& Cambon, Claude 2016 Spectral modelling for passive scalar dynamics in homogeneous anisotropic turbulence. Journal of Fluid Mechanics 799, 159-199.

Buaria, Dhawal, Pumir, Alain \& Bodenschatz, Eberhard 2020 Self-attenuation of extreme events in Navier-Stokes turbulence. Nature communications 11 (1), 1-7.

Burkovska, Olena, Glusa, Christian \& D'Elia, Marta 2020 An optimization-based approach to parameter learning for fractional type nonlocal models. arXiv preprint arXiv:2010.03666 .

Burton, Gregory C \& Dahm, Werner JA 2005 Multifractal subgrid-scale modeling for large-eddy simulation. II. Backscatter limiting and a posteriori evaluation. Physics of Fluids 17 (7), 075112.

Cairoli, Andrea 2016 Towards a Comprehensive Framework for the Analysis of Anomalous Diffusive Systems. PhD thesis, Queen Mary University of London.

Cerutti, Stefano, Meneveau, Charles \& Knio, Omar M 2000 Spectral and hyper eddy viscosity in high-Reynolds-number turbulence. Journal of Fluid Mechanics 421, 307-338.

Chao, Manuel Arias, Kulkarni, Chetan, Goebel, Kai \& Fink, Olga 2020 Fusing physics-based and deep learning models for prognostics. arXiv preprint arXiv:2003.00732 .

Chen, Hudong, Orszag, Steven A, Staroselsky, Ilya \& Succi, Sauro 2004 Expanded analogy between Boltzmann kinetic theory of fluids and turbulence. Journal of Fluid Mechanics 519, 301.

Deng, Weinua, Li, Buyang, Tian, Wenyi \& Zhang, Pingwen 2018 Boundary problems for the fractional and tempered fractional operators. Multiscale Modeling \& Simulation 16 (1), 125-149.

Di Leoni, P Clark, Zaki, Tamer A, Karniadakis, George \& Meneveau, Charles 2020 Two-point stress-strain rate correlation structure and non-local eddy viscosity in turbulent flows. arXiv preprint arXiv:2006.02280 .

Di Nezza, Eleonora, Palatucci, Giampiero \& Valdinoci, Enrico 2012 Hitchhiker's guide to the fractional Sobolev spaces. Bulletin des sciences mathématiques 136 (5), 521-573.

Egolf, Peter W \& Hutter, Kolumban 2017 Fractional Turbulence Models. Progress in Turbulence VII pp. 123-131.

Egolf, Peter William \& Hutter, K 2020 Nonlinear, Nonlocal and Fractional Turbulence. Graduate Studies in Mathematics. Springer, .

Epps, Brenden P \& Cushman-Roisin, Benoit 2018 Turbulence Modeling via the Fractional Laplacian. arXiv preprint arXiv:1803.05286.

Evin, G, Blanchet, J, Paquet, E, Garavaglia, F \& Penot, D 2016 A regional model for extreme rainfall based on weather patterns subsampling. Journal of Hydrology 541, 1185-1198.

Girimaji, Sharath S 2007 Boltzmann kinetic equation for filtered fluid turbulence. Physical review letters 99 (3), 034501.

Hamlington, Peter E \& Dahm, Werner JA 2008 Reynolds stress closure for nonequilibrium effects in turbulent flows. Physics of Fluids 20 (11), 115101.

Henderson, David W \& Taimina, Daina 2000 Experiencing geometry. Prentice Hall.

Hill, Reginald J 2002 Exact second-order structure-function relationships. Journal of Fluid Mechanics 468, 317-326.

Holgate, Joshua, Skillen, Alex, Craft, Timothy \& Revell, Alistair 2019 A review of embedded large eddy simulation for internal flows. Archives of Computational Methods in Engineering 26 (4), $865-882$.

Huang, LoRICK 2015 Density estimates for SDEs driven by tempered stable processes. arXiv preprint arXiv:1504.04183.

Ionescu, C, Lopes, A, Copot, Dana, Machado, JA Tenreiro \& Bates, JHT 2017 The role of fractional calculus in modeling biological phenomena: A review. Communications in Nonlinear Science and Numerical Simulation 51, 141-159.

Jacob, Jérôme, Malaspinas, Orestis \& Sagaut, Pierre 2018 A new hybrid recursive regularised Bhatnagar-Gross-Krook collision model for Lattice Boltzmann method-based large eddy simulation. Journal of Turbulence 19 (11-12), 1051-1076. 
Jin, Guodong, Wang, Shizhao, Wang, Yun \& He, Guowei 2018 Lattice Boltzmann simulations of highorder statistics in isotropic turbulent flows. Applied Mathematics and Mechanics 39 (1), 21-30.

Jouybari, Mostafa Aghaei, Yuan, Junlin, Brereton, Giles J \& Jaberi, Farhad A 2020 Supersonic turbulent channel flows over two and three dimensional sinusoidal rough walls. arXiv preprint arXiv:2012.02852 .

Kaleta, Kamil \& Sztonyk, Pawel 2015 Estimates of transition densities and their derivatives for jump Lévy processes. Journal of Mathematical Analysis and Applications 431 (1), 260-282.

Kharazmi, Ehsan \& Zayernouri, Mohsen 2019 Fractional sensitivity equation method: Application to fractional model construction. Journal of Scientific Computing 80 (1), 110-140.

Kurz, Marius \& Beck, ANdrea 2020 A machine learning framework for LES closure terms. arXiv preprint arXiv:2010.03030 .

Laval, JP, Dubrulle, B \& Nazarenko, S 2001 Nonlocality and intermittency in three-dimensional turbulence. Physics of Fluids 13 (7), 1995-2012.

Malaspinas, Orestis \& Sagaut, Pierre 2012 Consistent subgrid scale modelling for lattice Boltzmann methods. Journal of Fluid Mechanics 700, 514-542.

Meneveau, Charles 1994 Statistics of turbulence subgrid-scale stresses: Necessary conditions and experimental tests. Physics of Fluids 6 (2), 815-833.

Meral, FC, Royston, TJ \& Magin, R 2010 Fractional calculus in viscoelasticity: an experimental study. Communications in nonlinear science and numerical simulation 15 (4), 939-945.

Mishra, Aashwin Ananda \& Girimaji, Sharath 2019 Linear analysis of non-local physics in homogeneous turbulent flows. Physics of Fluids 31 (3), 035102.

Moser, Robert D, Haering, Sigfried W \& Yalla, Gopal R 2021 Statistical properties of subgrid-scale turbulence models. Annual Review of Fluid Mechanics 53.

Naghibolhosseini, Maryam \& Long, Glenis R 2018 Fractional-order modelling and simulation of human ear. International Journal of Computer Mathematics 95 (6-7), 1257-1273.

Pang, Guofei, D’Elia, Marta, Parks, Michael \& Karniadakis, George E 2020 nPinNs: nonlocal Physics-Informed Neural Networks for a parametrized nonlocal universal Laplacian operator. Algorithms and Applications. Journal of Computational Physics 422, 109760.

Patra, Abani K, Bevilacqua, Andrea \& Akhavan Safaei, Ali 2018 Analyzing complex models using data and statistics. In International conference on computational science, pp. 724-736. Springer.

Pawar, Suraj, San, Omer, Rasheed, Adil \& Vedula, Prakash 2020 A priori analysis on deep learning of subgrid-scale parameterizations for Kraichnan turbulence. Theoretical and Computational Fluid Dynamics 34 (4), 429-455.

Piomelli, U 2014 Large eddy simulations in 2030 and beyond. Philosophical Transactions of the Royal Society A: Mathematical, Physical and Engineering Sciences 372 (2022), 20130320.

Pope, Stephen B 2001 Turbulent flows.

Portwood, G. D., Nadiga, B. T., Saenz, J. A. \& Livescu, D. 2021 Interpreting neural network models of residual scalar flux. Journal of Fluid Mechanics 907, A23.

Premnath, Kannan N, Pattison, Martin J \& Banerjee, Sanjoy 2009 Dynamic subgrid scale modeling of turbulent flows using lattice-Boltzmann method. Physica A: Statistical Mechanics and its Applications 388 (13), 2640-2658.

Sabzikar, Farzad, Meerschaert, Mark M \& Chen, Jinghua 2015 Tempered fractional calculus. Journal of Computational Physics 293, 14-28.

Sagaut, Pierre 2010 Toward advanced subgrid models for Lattice-Boltzmann-based Large-eddy simulation: theoretical formulations. Computers \& Mathematics with Applications 59 (7), 2194-2199.

Sagaut, Pierre \& Cambon, Claude 2008 Homogeneous turbulence dynamics, , vol. 10. Springer.

Samiee, Mehdi, Akhavan-Safaei, Ali \& Zayernouri, Mohsen $2020 a$ A fractional subgrid-scale model for turbulent flows: Theoretical formulation and a priori study. Physics of Fluids 32 (5), 055102.

Samiee, Mehdi, Kharazmi, Ehsan, Meerschaert, Mark M \& Zayernouri, Mohsen $2020 b$ A Unified Petrov-Galerkin Spectral Method and Fast Solver for Distributed-Order Partial Differential Equations. Communications on Applied Mathematics and Computation pp. 1-30.

Samiee, Mehdi, Zayernouri, Mohsen \& Meerschaert, Mark M 2019 A unified spectral method for FPDEs with two-sided derivatives; part I: a fast solver. Journal of Computational Physics 385, 225-243.

She, Zhen-Su, Jackson, Eric \& Orszag, Steven A 1990 Intermittent vortex structures in homogeneous isotropic turbulence. Nature 344 (6263), 226-228. 
She, Zhen-Su \& Leveque, Emmanuel 1994 Universal scaling laws in fully developed turbulence. Physical review letters 72 (3), 336.

Shivamoggi, BK \& Tuovila, N 2019 Direct interaction approximation for non-Markovianized stochastic models in the turbulence problem. Chaos: An Interdisciplinary Journal of Nonlinear Science 29 (6), 063124 .

Sirignano, Justin, MacArt, Jonathan F \& Freund, Jonathan B 2020 DPM: A deep learning PDE augmentation method with application to large-eddy simulation. Journal of Computational Physics 423, 109811.

SMAGORINSKy, JoSEPH 1963 General circulation experiments with the primitive equations: I. The basic experiment. Monthly weather review 91 (3), 99-164.

Soto, Rodrigo 2016 Kinetic theory and transport phenomena, , vol. 25. Oxford University Press.

Stein, Elias M 1970 Singular integrals and differentiability properties of functions, , vol. 2. Princeton university press.

Suzuki, Jorge, Zhou, Yongtao, D’Elia, Marta \& Zayernouri, Mohsen 2021 A thermodynamically consistent fractional visco-elasto-plastic model with memory-dependent damage for anomalous materials. Computer Methods in Applied Mechanics and Engineering 373, 113494.

Suzuki, Jorge L, Varghaei, Pegah, Kharazmi, Ehsan \& Zayernouri, Mohsen 2020 Anomalous Nonlinear Dynamics Behavior of Fractional Viscoelastic Structures. arXiv preprint arXiv:2009.12214

Suzuki, Jorge L \& Zayernouri, Mohsen 2020 A self-singularity-capturing scheme for fractional differential equations. International Journal of Computer Mathematics pp. 1-28.

Taghizadeh, Salar, Witherden, Freddie D \& Girimaji, Sharath S 2020 Turbulence closure modeling with data-driven techniques: physical compatibility and consistency considerations. New Journal of Physics 22 (9), 093023.

Vincent, Albert \& Meneguzzi, Maria 1991 The spatial structure and statistical properties of homogeneous turbulence. Journal of Fluid Mechanics 225, 1-20.

Weron, Rafat 2001 Levy-stable distributions revisited: tail index> 2 does not exclude the Levy-stable regime. International Journal of Modern Physics C 12 (02), 209-223.

Willard, Jared, Jia, Xiaowei, Xu, Shaoming, Steinbach, Michael \& Kumar, Vipin 2020 Integrating physics-based modeling with machine learning: A survey. arXiv preprint arXiv:2003.04919 .

XIE, Changping \& FAng, Shaomei 2019 A second-order finite difference method for fractional diffusion equation with Dirichlet and fractional boundary conditions. Numerical Methods for Partial Differential Equations 35 (4), 1383-1395.

YANG, XIA \& LozANo-Durán, A 2017 A multifractal model for the momentum transfer process in wallbounded flows. Journal of Fluid Mechanics 824.

You, Huaigian, Yu, Yue, Trask, Nathaniel, Gulian, Mamikon \& D’Elia, Marta 2021 Data-driven learning of nonlocal physics from high-fidelity synthetic data. Computer Methods in Applied Mechanics and Engineering 374, 113553.

Zaky, Mahmoud A, Hendy, Ahmed S \& Macías-Díaz, Jorge E 2020 Semi-implicit Galerkin-Legendre spectral schemes for nonlinear time-space fractional diffusion-reaction equations with smooth and nonsmooth solutions. Journal of Scientific Computing 82 (1), 1-27.

Zayernouri, Mohsen, Ainsworth, Mark \& Karniadakis, George Em 2015 Tempered Fractional SturmLiouville EigenProblems. SIAM Journal on Scientific Computing 37 (4), A1777-A1800.

Zayernouri, Mohsen \& Karniadakis, George Em 2013 Fractional Sturm-Liouville eigen-problems: theory and numerical approximation. Journal of Computational Physics 252, 495-517.

Zhang, Zhijiang, Deng, Weihua \& Karniadakis, George Em 2018 a Riesz basis Galerkin method for the tempered fractional Laplacian. SIAM Journal on Numerical Analysis 56 (5), 3010-3039.

ZhiYin, YANg 2015 Large-eddy simulation: Past, present and the future. Chinese journal of Aeronautics 28 (1), 11-24. 